\title{
Non-trapping estimates near normally hyperbolic trapping
}

\author{
Peter Hintz And Andras Vasy
}

In this paper, we prove semiclassical resolvent estimates for operators with normally hyperbolic trapping which are lossless relative to non-trapping estimates but take place in weaker function spaces. In particular, we obtain non-trapping estimates in standard $L^{2}$ spaces for the resolvent sandwiched between operators which localize away from the trapped set $\Gamma$ in a rather weak sense, namely whose principal symbols vanish on $\Gamma$.

\section{Introduction}

The purpose of this paper is to obtain semiclassical estimates for pseudodiffererential operators $P_{h}(z)$ with normally hyperbolic trapping for $z$ real which are lossless relative to non-trapping estimates, but take place in weaker function spaces which are defined in a manner related to the Hamiltonian dynamics. Thus, the main result is an estimate of the form

$$
\|u\|_{\mathcal{H}_{h, \Gamma}} \leq C h^{-1}\left\|P_{h}(z) u\right\|_{\mathcal{H}_{h, \Gamma}^{*}},
$$

with certain function spaces $\mathcal{H}_{h, \Gamma}$ and $\mathcal{H}_{h, \Gamma}^{*}$, described below; away from the trapped set these are just standard $L^{2}$ spaces. As the main application of such estimates is in so-called b-spaces, e.g., Kerr-de Sitter spaces, for which the estimates follow from the semiclassical ones immediately in the presence of dilation invariance, we also prove their counterpart in the general, non-dilation-invariant, b-setting. Extensions of special cases of these estimates play an important role in the recent global analysis of nonlinear wave equations on asymptotically Kerr-de Sitter spaces by the authors [6].

So at first we consider a family $P_{h}(z)$ of semiclassical pseudodifferential operators $P_{h}(z) \in \Psi_{h}(X)$ on a closed manifold $X$, depending smoothly on the parameter $z \in \mathbb{C}$, with normally hyperbolic trapping at the trapped set $\Gamma$, and assume that $P_{h}(z)$ is formally self-adjoint near $\Gamma$ for $z \in \mathbb{R}$; moreover, we add complex absorption $W$ in such a way that all forward and backward 
bicharacteristics outside $\Gamma$ either enter the elliptic set of $W$ in finite time or tend to $\Gamma$, and in at least one of the two directions they tend to the elliptic set of $W$. The bicharacteristics tending to $\Gamma$ in the forward/backward directions are forward/backward trapped; denote by $\Gamma_{-}$, resp. $\Gamma_{+}$the forward, resp. backward trapped set, ${ }^{1}$ and assume that these are smooth codimension one submanifolds of $T^{*} X$ which intersect transversally in $\Gamma$, which we moreover assume to be symplectic.

In this normally hyperbolic setting, under additional hypotheses, Wunsch and Zworski [12] have shown polynomial semiclassical resolvent estimates

$$
\|u\| \leq C h^{-N}\left\|P_{h}(z) u\right\|, 0<h<h_{0},
$$

in small strips $|\operatorname{Im} z| \leq c h, c>0$ sufficiently small, $N>1$, and indeed for $z$ real, the loss (as compared to non-trapping estimates, which hold in many cases where there is no trapping, and which lose a power of $h^{-1}$ ) is merely logarithmic, i.e., one has

$$
\|u\| \leq C h^{-1}\left(\log h^{-1}\right)\left\|P_{h}(z) u\right\|, 0<h<h_{0},
$$

where $\|\cdot\|$ is the $L^{2}$-norm; Bony, Burq and Ramond [1] showed that (1.2) is indeed sharp. Dyatlov [5] improved these estimates in $\operatorname{Im} z<0$ by making $c$ and $N$ explicit; in a more general setting, Nonnenmacher and Zworski [9] obtained the optimal value for $c$.

We are concerned with improved estimates (for $z$ almost real) if one localizes $u$ and $P_{h}(z) u$ away from the trapping $\Gamma$ in a rather weak sense, such as by applying pseudodifferential operators with symbols vanishing at $\Gamma$. To place this into context, recall that Datchev and Vasy $[2,3]$ have shown that under our assumptions, with $\operatorname{Im} z=\mathcal{O}\left(h^{\infty}\right)$, if $A, B \in \Psi_{h}(X)$ with $\mathrm{WF}_{h}^{\prime}(A) \cap \Gamma=\mathrm{WF}_{h}^{\prime}(B) \cap \Gamma=\emptyset, B$ elliptic on $\mathrm{WF}_{h}^{\prime}(A)$, then for all $M$ there is $N$ such that

$$
\|A u\| \leq C h^{-1}\left\|B P_{h}(z) u\right\|+C^{\prime} h^{M}\|u\|+C^{\prime \prime} h^{-N}\left\|(\operatorname{Id}-B) P_{h}(z) u\right\| .
$$

Thus, if $P_{h}(z) u$ is $\mathcal{O}\left(h^{N-1}\right)$ at $\Gamma$ (corresponding to the $\operatorname{Id}-B$ term in the estimate), then on the elliptic set of $A$, hence off $\Gamma$ by appropriate choice

\footnotetext{
${ }^{1}$ In the notation of Wunsch and Zworski [12], which we recall below in Section 2.1, $\Gamma_{ \pm}$are the backward/forward trapped sets for all (not necessarily null) bicharacteristics near the, say, zero level set of the semiclassical principal symbol $p_{h, z}$, and $\Gamma_{ \pm}^{\lambda}$ are the corresponding sets within the $\lambda$-level set of $p_{h, z}$.
} 
of $A, u$ satisfies non-trapping semiclassical estimates:

$$
\left\|A P_{h}(z)^{-1} A v\right\| \leq C h^{-1}\|v\|
$$

with $A$ as above (take $B$ as above with $\left.\mathrm{WF}_{h}^{\prime}(\mathrm{Id}-B) \cap \mathrm{WF}_{h}^{\prime}(A)=\emptyset\right)$. Here the $\mathcal{O}\left(h^{\infty}\right)$ bound on $\operatorname{Im} z$ arises from the a priori estimate, (1.1), and if $1<N<3 / 2$, e.g., as is on, and sufficiently near, the real axis, ${ }^{2}$ then one can take $\operatorname{Im} z=\mathcal{O}\left(h^{-1+2 N}\right)$. The purpose of this paper is to improve this result by relaxing the conditions on $\mathrm{WF}_{h}^{\prime}(A)$ and $\mathrm{WF}_{h}^{\prime}(B)$ in (1.3).

The main point of the theorem below is thus that its estimate degenerates only at, as opposed to near, $\Gamma$. The proof given here is closely related to the proof of Wunsch and Zworski [12, Section 4] but can take place in a significantly simpler, standard semiclassical pseudodifferential algebra, at the cost of being suboptimal in terms of the $L^{2}$-estimate, even though it is optimal (i.e., non-trapping) when a pseudodifferential operator with vanishing principal symbol at $\Gamma$ is applied from both sides. To set this up, let $Q_{ \pm} \in \Psi_{h}^{-\infty}(X)$ be self-adjoint and have symbols which are defining functions of $\Gamma_{ \pm}$near $\Gamma$, say on a neighborhood $O$ of $\Gamma$. Let $Q_{0} \in \Psi_{h}^{0}(X)$ be a semiclassical operator with $\mathrm{WF}_{h}^{\prime}\left(Q_{0}\right) \cap \Gamma=\emptyset$ which is elliptic on $O^{c}$ (and thus on a neighborhood of $O^{c}$ ), with real principal symbol for convenience. One considers normally isotropic spaces at $\Gamma$, denoted $\mathcal{H}_{h, \Gamma}$, with squared norms given by

$$
\|u\|_{\mathcal{H}_{h, \Gamma}}^{2}=\left\|Q_{0} u\right\|^{2}+\left\|Q_{+} u\right\|^{2}+\left\|Q_{-} u\right\|^{2}+h\|u\|^{2}
$$

this is just the standard $L^{2}$-space microlocally away from $\Gamma$ as one of $Q_{+}$, $Q_{-}$or $Q_{0}$ is elliptic there, and it does not depend on the choice of $Q_{0}$ as on $O \backslash \Gamma$ one of $Q_{+}$and $Q_{-}$is elliptic at every point. The dual space relative to $L^{2}$ is then ${ }^{3}$

$$
\mathcal{H}_{h, \Gamma}^{*}=h^{1 / 2} L^{2}+Q_{+} L^{2}+Q_{-} L^{2}+Q_{0} L^{2}
$$

(which is $L^{2}$ as a space, but with this norm); $P_{h}(z) u$ will then be measured in $\mathcal{H}_{h, \Gamma}^{*}$.

\footnotetext{
${ }^{2}$ In the latter case by the Phragmén-Lindelöf theorem.

${ }^{3}$ One really has $Q_{ \pm}^{*}$ and $Q_{0}^{*}$ in this formula, but the reality of the principal symbols assures that one may replace them by $Q_{ \pm}$and $Q_{0}$ modulo $h L^{2}$. See $[8$, Appendix A] for a general discussion of the underlying functional analysis; also see Footnote 11.
} 
Theorem 1.1. Let $P=P_{h}(z), Q_{ \pm}$be as above, $\operatorname{Im} z=\mathcal{O}\left(h^{2}\right)$. Then

$$
\left\|Q_{+} u\right\|+\left\|Q_{-} u\right\| \leq C h^{-1}\|P u\|_{\mathcal{H}_{h, \Gamma}^{*}}+C^{\prime} h^{1 / 2}\|u\|
$$

and thus by (1.2),

$$
\|u\|_{\mathcal{H}_{h, \Gamma}} \leq C h^{-1}\|P u\|_{\mathcal{H}_{h, \Gamma}^{*}} .
$$

In fact, we also obtain a direct proof of (1.5) without using (1.2) at the end of Section 2. Note that this theorem in particular implies the main result of [2] in this setting, in that the estimates are of the same kind, except that in [2] $P u$ is assumed to be microlocalized away from $\Gamma$, and $u$ is estimated microlocally away from $\Gamma$.

The aforementioned b-estimates will be proved in Section 3, see Theorem 3.2.

\section{Semiclassical resolvent estimates on the real line}

\subsection{Notation and definitions}

We will review some definitions of semiclassical analysis, partially in order to fix our notation. For a general reference, see Zworski [13].

Let $X$ be a compact $n$-dimensional manifold without boundary, and fix a smooth density on $X$.

- For $u \in L^{2}(X)$, denote by $\|u\|$ its $L^{2}(X)$ norm; moreover, denote by $\langle\cdot, \cdot\rangle$ the (sesquilinear) inner product on $L^{2}(X)$.

- A family of functions $u=\left(u_{h}\right)_{h \in(0,1)}$ on $X$ is polynomially bounded if $\|u\| \leq C h^{-N}$ for some $N$. If $k \in \mathbb{R}$, we say that $u \in \mathcal{O}\left(h^{k}\right)$ if $\|u\| \leq$ $C_{k} h^{k}$, and $u \in \mathcal{O}\left(h^{\infty}\right)$ if $\|u\| \leq C_{N} h^{N}$ for every $N$.

- For $a=\left(a_{h}\right)_{h \in(0,1)} \in \mathcal{C}^{\infty}\left(T^{*} X\right)$, we say $a \in h^{k} S^{m}\left(T^{*} X\right)$ if $a$ satisfies

$$
\left|\partial_{z}^{\alpha} \partial_{\zeta}^{\beta} a_{h}(z, \zeta)\right| \leq C_{\alpha \beta} h^{k}\langle\zeta\rangle^{m-|\beta|}
$$

for all multiindices $\alpha, \beta$ and all $N \in \mathbb{N}$ in any coordinate chart, where the $z$ are coordinates in the base and $\zeta$ coordinates in the fiber. We define the semiclassical quantization $\mathrm{Op}_{h}(a)$ of $a$ by

$$
\mathrm{Op}_{h}(a) u(z)=(2 \pi h)^{-n} \int e^{i z \zeta / h} a(z, \zeta) \hat{u}(\zeta / h) d \zeta
$$


for $u \in \mathcal{C}_{\mathrm{c}}^{\infty}(X)$ supported in a chart and for general $u \in \mathcal{C}_{\mathrm{c}}^{\infty}(X)$ by using a partition of unity. We write $\mathrm{Op}_{h}(a) \in h^{k} \Psi_{h}^{m}(X)$. The quantization depends on the choice of partition of unity, but the resulting class of operators does not, modulo operators that have Schwartz kernel in $h^{\infty} \mathcal{C}^{\infty}\left(X^{2}\right)$. We say that $a$ is a symbol of $\operatorname{Op}_{h}(a)$. The equivalence class of $a$ in $h^{k} S^{m}\left(T^{*} X\right) / h^{k-1} S^{m-1}\left(T^{*} X\right)$ is invariantly defined and is called the principal symbol of $\mathrm{Op}_{h}(a)$. All operators below except $Q_{0} \in \Psi_{h}^{0}(X)$ will in fact have compact microsupport in the sense that they are quantizations of symbols $a \in h^{k} S^{m}\left(T^{*} X\right)$ satisfying in addition for all $N$

$$
\left|\partial_{z}^{\alpha} \partial_{\zeta}^{\beta} a_{h}(z, \zeta)\right| \leq C_{N} h^{N}\langle\zeta\rangle^{-N} \text { for all multiindices } \alpha, \beta
$$

for $\zeta$ outside of a compact subset of $T^{*} X$. We denote the class of such symbols by $h^{k} S\left(T^{*} X\right)$ and the corresponding class of operators by $h^{k} \Psi_{h}(X)$.

- If $A, B \in \Psi_{h}(X)$, then $[A, B] \in h \Psi_{h}(X)$, and its principal symbol is $\frac{h}{i} \mathrm{H}_{a} b$, where we define the Hamilton vector field in a coordinate chart by

$$
\mathrm{H}_{a}=\left(\partial_{\zeta} a\right) \partial_{z}-\left(\partial_{z} a\right) \partial_{\zeta}
$$

- By a bicharacteristic of $A$ we mean an integral curve of the Hamilton vector field of the principal symbol of $A$. We denote the integral curve passing through the point $\rho \in T^{*} X$ by $\gamma_{\rho}$, i.e., $\gamma_{\rho}(0)=\rho$ and $\gamma_{\rho}^{\prime}(s)=$ $\mathrm{H}_{a}\left(\gamma_{\rho}(s)\right)$. We shall also write $\phi^{s}(\rho):=\gamma_{\rho}(s)$ for the bicharacteristic flow.

- For a polynomially bounded family $\left(u_{h}\right)_{h \in(0,1)}$ and $k \in \mathbb{R} \cup\{\infty\}$, we say that $u=\mathcal{O}\left(h^{k}\right)$ at a point $\rho \in T^{*} X$ if there exists $a \in S\left(T^{*} X\right)$ with $a(\rho) \neq 0$ such that $\left\|\mathrm{Op}_{h}(a) u\right\|=\mathcal{O}\left(h^{k}\right)$. We define the semiclassical wave front set $\mathrm{WF}_{h}(u)$ of $u$ as the complement of the set of all $\rho \in T^{*} X$ at which $u=\mathcal{O}\left(h^{\infty}\right)$.

- The microsupport of $A=\mathrm{Op}_{h}(a) \in h^{k} \Psi_{h}(X)$, denoted $\mathrm{WF}_{h}^{\prime}(A)$, is the complement of the set of all $\rho \in T^{*} X$ so that $\left|\partial^{\alpha} a\right|=\mathcal{O}\left(h^{\infty}\right)$ near $\rho$ for every multiindex $\alpha$, in any (and therefore in every) coordinate chart.

- For $A \in h^{k} \Psi_{h}(X)$ with principal symbol $a \in h^{k} S\left(T^{*} X\right)$, we say that $A$ is elliptic at $\rho \in T^{*} X$ if there is a constant $C>0$ such that $\left|a\left(\rho^{\prime}\right)\right| \geq$ $C h^{k}$ for $\rho^{\prime}$ near $\rho$ and $h$ sufficiently small. For a subset $E \subset T^{*} X$, we say that $A$ is elliptic on $E$ if $A$ is elliptic at each point of $E$. If $A \in$ 
$h^{k} \Psi_{h}(X)$ is elliptic on $E \subset T^{*} X$ and $A u=f$ with $u, f$ polynomially bounded and $f$ is $\mathcal{O}(1)$ on $E$, then microlocal elliptic regularity states that $u$ is $\mathcal{O}\left(h^{-k}\right)$ on $E$.

- The semiclassical characteristic set of the semiclassical operator $A \in$ $\Psi_{h}(X)$ with principal symbol $a$ is defined by $\Sigma_{h}=\left\{\rho \in T^{*} X: a(\rho)=\right.$ $0\}$.

- If $A \in \Psi_{h}(X)$ has a principal symbol with non-positive imaginary part, $u, f$ are polynomially bounded, $A u=f$, and $u=\mathcal{O}\left(h^{k}\right)$ at $\rho$, $f=\mathcal{O}\left(h^{k+1}\right)$ on $\gamma_{\rho}([0, T])$ for some $T>0$, then the propagation of singularities states that $u=\mathcal{O}\left(h^{k}\right)$ at $\gamma_{\rho}(T)$.

- Let $P \in \Psi_{h}(X)$ be a semiclassical operator. Let $U \subset X$ denote an open subset so that the cotangent bundle over $U$ contains what will be the trapped set, and place complex absorbing potentials in a neighborhood of $U^{c}$. ${ }^{4}$ We recall the notion of normal hyperbolicity from [12]: Define the backward, resp. forward, trapped set $\Gamma_{+}$, resp. $\Gamma_{-}$, by

$$
\Gamma_{ \pm}=\left\{\rho \in T^{*} X: \gamma_{\rho}(s) \notin T_{U^{c}}^{*} X \text { for all } \mp s \geq 0\right\}
$$

Let $\Gamma_{ \pm}^{\lambda}=\Gamma_{ \pm} \cap p^{-1}(\lambda)$ be the backward/forward trapped set within the energy surface $p^{-1}(\lambda)$, and define the trapped set $\Gamma_{\lambda}:=\Gamma_{+}^{\lambda} \cap \Gamma_{-}^{\lambda}$. We say that $P$ is normally hyperbolically trapping if:

(1) There exists $\delta>0$ such that $d p \neq 0$ on $p^{-1}(\lambda)$ for $|\lambda|<\delta$;

(2) $\Gamma_{ \pm} \cap p^{-1}(-\delta, \delta)$ are smooth codimension one submanifolds intersecting transversally at $\Gamma \cap p^{-1}(-\delta, \delta)$, and $\Gamma \cap p^{-1}(-\delta, \delta)$ is symplectic;

(3) the flow is hyperbolic in the normal directions to $\Gamma_{\lambda}$ within the energy surface: There exist subbundles $E_{\lambda}^{ \pm}$of $T_{\Gamma_{\lambda}}\left(\Gamma_{ \pm}^{\lambda}\right)$ such that $T_{\Gamma_{\lambda}} \Gamma_{ \pm}^{\lambda}=$ $T \Gamma_{\lambda} \oplus E_{\lambda}^{ \pm}$, where $d \phi^{s}: E_{\lambda}^{ \pm} \rightarrow E_{\lambda}^{ \pm}$, and there exists $\theta>0$ such that for all $|\lambda|<\delta$

$$
\left\|d \phi^{s}(v)\right\| \leq C e^{-\theta|t|}\|v\| \text { for all } v \in E_{\lambda}^{\mp}, \pm t \geq 0
$$

\footnotetext{
${ }^{4}$ See $[10,12]$ for details; the point here is that the relevant part of our analysis takes place microlocally near the trapped set, and the complex absorbing potentials allow us to "cut off" the bicharacteristic flow in a neighborhood of the trapped set.
} 


\subsection{Details on the setup and proof of the main result}

Let $p=p_{h, z}$ be the semiclassical principal symbol of $P=P_{h}(z)$. Recall from the work of Wunsch and Zworski [12, Lemma 4.1], with a corrected argument in [11], that for defining functions $\phi_{ \pm}$of $\Gamma_{ \pm}$(near $\Gamma$, namely in a neighborhood $O$ of $\Gamma$ ) one can take $\phi_{ \pm}$with

$$
\mathrm{H}_{p} \phi_{ \pm}=\mp c_{ \pm}^{2} \phi_{ \pm}
$$

with $c_{ \pm}>0$ near $\Gamma$, and with ${ }^{5}$

$$
\left\{\phi_{+}, \phi_{-}\right\}>0
$$

near $\Gamma$. This is the only relevant feature of normal hyperbolicity for this paper; thus these identities and estimates could be taken as its definition for our purposes. By shrinking $O$ if necessary we may assume that this Poisson bracket as well as $c_{ \pm}$have positive lower bounds on $O$. Then notice that

$$
\mathrm{H}_{p} \phi_{+}^{2}=-2 c_{+}^{2} \phi_{+}^{2}, \quad \mathrm{H}_{p} \phi_{-}^{2}=2 c_{-}^{2} \phi_{-}^{2} .
$$

As indicated in the introduction, we consider normally isotropic spaces at $\Gamma$, denoted $\mathcal{H}_{h, \Gamma}$, with squared norms given by

$$
\|u\|_{\mathcal{H}_{h, \Gamma}}^{2}=\left\|Q_{0} u\right\|^{2}+\left\|Q_{+} u\right\|^{2}+\left\|Q_{-} u\right\|^{2}+h\|u\|^{2}
$$

we can take $Q_{ \pm}$with principal symbol $\phi_{ \pm}$, while $Q_{0}$ is elliptic on $O^{c}$ with real principal symbol. This is just the standard $L^{2}$-space microlocally away from $\Gamma$ as one of $Q_{+}, Q_{-}$and $Q_{0}$ is elliptic there, and it does not depend on the choice of $Q_{0}$ as on $O \backslash \Gamma$ one of $Q_{+}$and $Q_{-}$is elliptic at every point. Notice that in fact

$$
\left(Q_{+}-i Q_{-}\right)^{*}\left(Q_{+}-i Q_{-}\right)=Q_{+}^{*} Q_{+}+Q_{-}^{*} Q_{-}-i\left[Q_{+}, Q_{-}\right]
$$

and if $B \in \Psi_{h}(X)$ with $\mathrm{WF}_{h}^{\prime}(B) \subset O$ then

$$
h\|B v\|^{2} \leq C \operatorname{Re}\left\langle i\left[Q_{+}, Q_{-}\right] B v, B v\right\rangle+C h^{N^{\prime}}\|v\|^{2},
$$

\footnotetext{
${ }^{5}$ These defining functions exist globally when $\Gamma_{ \pm}$is orientable; but even if $\Gamma_{ \pm}$ is not such, the square is globally defined. There is only a minor change required below if $\phi_{ \pm}$are not well defined; see Footnote 6 .
} 
$C>0$, in view of $\left\{\phi_{+}, \phi_{-}\right\}>0$ on $O$, so

$$
\begin{aligned}
& Q_{+}^{*} Q_{+}+Q_{-}^{*} Q_{-} \\
& \quad=\frac{1}{2}\left(Q_{+}^{*} Q_{+}+Q_{-}^{*} Q_{-}+\left(Q_{+}-i Q_{-}\right)^{*}\left(Q_{+}-i Q_{-}\right)+i\left[Q_{+}, Q_{-}\right]\right)
\end{aligned}
$$

shows that, for $h>0$ small, the norm on $\mathcal{H}_{h, \Gamma}$ is equivalent to just the norm

$$
\|u\|_{\mathcal{H}_{h, \Gamma}, 2}^{2}=\left\|Q_{0} u\right\|^{2}+\left\|Q_{+} u\right\|^{2}+\left\|Q_{-} u\right\|^{2}
$$

As mentioned in the introduction, the dual space relative to $L^{2}$ is then

$$
\mathcal{H}_{h, \Gamma}^{*}=h^{1 / 2} L^{2}+Q_{+} L^{2}+Q_{-} L^{2}+Q_{0} L^{2} .
$$

Then $\Psi_{h}(X)$ acts on $\mathcal{H}_{h, \Gamma}$, and thus on $\mathcal{H}_{h, \Gamma}^{*}$, for $B \in \Psi_{h}(X)$ preserves $h^{-1 / 2} L^{2}$ and gives

$$
\left\|Q_{+} B u\right\| \leq\left\|B Q_{+} u\right\|+\left\|\left[Q_{+}, B\right] u\right\| \leq C\left\|Q_{+} u\right\|+h\|u\|_{L^{2}},
$$

with a similar result for $Q_{-}$and $Q_{0}$. We remark that the notation $\mathcal{H}_{h, \Gamma}$ is justified as the space depends only on $\Gamma$, not on the particular defining functions $\phi_{ \pm}$as any other defining functions would change $Q_{ \pm}$by an elliptic factor modulo an element of $h \Psi_{h}(X)$, whose contribution to the squared norm can be absorbed into $C h^{2}\|u\|_{L^{2}}^{2}$, and thus dropped altogether (for $h$ small) in view of the equivalence of the two norms discussed above.

We are now ready to prove Theorem 1.1 . We remark that the microlocal version of the two estimates of the theorem is that given any neighborhood $O^{\prime}$ of $\Gamma$ with closure in $O$, there exist $B_{0} \in \Psi_{h}(X)$ elliptic at $\Gamma, B_{1}, B_{2} \in \Psi_{h}(X)$ with $\mathrm{WF}_{h}^{\prime}\left(B_{2}\right) \cap \Gamma_{+}=\emptyset, \mathrm{WF}_{h}^{\prime}\left(B_{j}\right) \subset O^{\prime}$ for $j=0,1,2$ such that

$$
\begin{aligned}
\left\|B_{0} Q_{+} u\right\|+\left\|B_{0} Q_{-} u\right\| \leq & h^{-1}\left\|B_{1} P u\right\|_{\mathcal{H}_{h, \Gamma}^{*}}+\left\|B_{2} u\right\|_{L^{2}} \\
& +C^{\prime} h^{1 / 2}\|u\|_{L^{2}}
\end{aligned}
$$

respectively

$$
\left\|B_{0} u\right\|_{\mathcal{H}_{h, \Gamma}} \leq h^{-1}\left\|B_{1} P u\right\|_{\mathcal{H}_{h, \Gamma}^{*}}+\left\|B_{2} u\right\|_{L^{2}}+C^{\prime} h\|u\|_{L^{2}}
$$

see (2.9). The theorem is then proved by controlling the $B_{2} u$ term using the backward non-trapped nature of $\Gamma_{-} \backslash \Gamma$.

Proof of Theorem 1.1. We first prove (1.4), which proves (1.5) by (1.2). In fact, one can also give a direct proof of (1.5) without using (1.2); see the discussion following this proof. 
Let $\chi_{0}(t)=e^{-1 / t}$ for $t>0, \chi_{0}(t)=0$ for $t \leq 0, \quad \chi \in \mathcal{C}_{c}^{\infty}([0, \infty))$ be identically 1 near 0 with $\chi^{\prime} \leq 0$, and indeed with $\chi^{\prime} \chi=-\chi_{1}^{2}, \chi_{1} \geq 0, \chi_{1} \in$ $\mathcal{C}_{c}^{\infty}([0, \infty))$, and let $\psi \in \mathcal{C}_{c}^{\infty}(\mathbb{R})$ be identically 1 near 0 . Let

$$
a=\chi_{0}\left(\phi_{+}^{2}-\phi_{-}^{2}+\kappa\right) \chi\left(\phi_{+}^{2}\right) \psi(p),
$$

$\kappa>0$ small. Notice that on $\operatorname{supp} a$, if $\chi$ is supported in $[0, R]$,

$$
\phi_{+}^{2} \leq R, \phi_{-}^{2} \leq \phi_{+}^{2}+\kappa=R+\kappa,
$$

so $a$ is localized near $\Gamma$ if $R$ and $\kappa$ are taken sufficiently small. Then

$$
\begin{aligned}
\frac{1}{4} \mathrm{H}_{p}\left(a^{2}\right)= & -\left(c_{+}^{2} \phi_{+}^{2}+c_{-}^{2} \phi_{-}^{2}\right)\left(\chi_{0} \chi_{0}^{\prime}\right)\left(\phi_{+}^{2}-\phi_{-}^{2}+\kappa\right) \chi\left(\phi_{+}^{2}\right)^{2} \psi(p)^{2} \\
& -c_{+}^{2} \phi_{+}^{2}\left(\chi^{\prime} \chi\right)\left(\phi_{+}^{2}\right) \chi_{0}\left(\phi_{+}^{2}-\phi_{-}^{2}+\kappa\right)^{2} \psi(p)^{2} .
\end{aligned}
$$

Now $\chi_{0}^{\prime} \geq 0$, so the two terms have opposite signs. Let $^{6}$

$$
a_{ \pm}=\phi_{ \pm} \sqrt{\left(\chi_{0} \chi_{0}^{\prime}\right)\left(\phi_{+}^{2}-\phi_{-}^{2}+\kappa\right)} \chi\left(\phi_{+}^{2}\right) \psi(p),
$$

and

$$
e_{-}=c_{+} \phi_{+} \chi_{1}\left(\phi_{+}^{2}\right) \chi_{0}\left(\phi_{+}^{2}-\phi_{-}^{2}+\kappa\right) \psi(p)
$$

then

$$
\frac{1}{4} \mathrm{H}_{p}\left(a^{2}\right)=-c_{+}^{2} a_{+}^{2}-c_{-}^{2} a_{-}^{2}+e_{-}^{2}
$$

Here

$$
\begin{aligned}
& \operatorname{supp} e_{-} \subset \operatorname{supp} a \\
& \operatorname{supp} e_{-} \cap \Gamma_{+}=\emptyset
\end{aligned}
$$

with the last statement following from $\phi_{+}^{2}$ taking values away from 0 on $\operatorname{supp} \chi_{1}$; see Figure 1 .

One then takes $A \in \Psi_{h}(X)$ with principal symbol $a$, and with $\mathrm{WF}_{h}^{\prime}(A) \subset$ supp $a, A_{ \pm} \in \Psi_{h}(X)$ with principal symbols of $a_{ \pm}$, and with $\mathrm{WF}_{h}^{\prime}\left(A_{ \pm}\right) \subset$ supp $a_{ \pm}, C_{ \pm}$have symbol $c_{ \pm}$and with $\mathrm{WF}_{h}^{\prime}\left(C_{ \pm}\right) \subset \operatorname{supp} c_{ \pm}$; one similarly

\footnotetext{
${ }^{6}$ If $\phi_{ \pm}$is not defined globally, $a_{ \pm}$are not defined as stated. (The term $e_{-}^{2}$ need not have a sign, so this issue does not arise for it; see the Weyl quantization argument below.) However, $a_{ \pm}$need not be real below, so as long as one can choose $\psi_{ \pm}$ complex valued with $\left|\psi_{ \pm}\right|^{2}=\phi_{ \pm}^{2}$, replacing the first factor of $\phi_{ \pm}$with $\psi_{ \pm}$in the definition of $a_{ \pm}$allows one to complete the argument in general.
} 


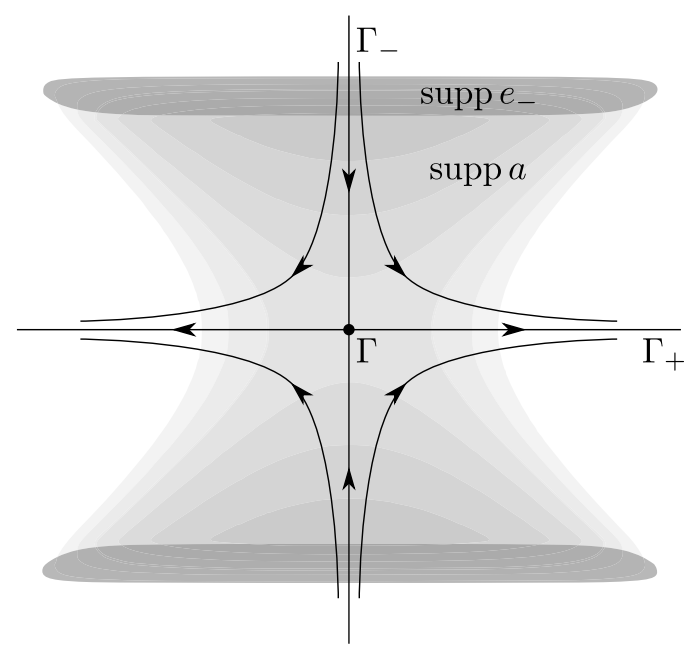

Figure 1: Supports of the commutant $a$ and the error term $e_{-}$in the positive commutator argument of the non-trapping estimate near the trapped set $\Gamma$, Theorem 1.1. The support of $a$ is indicated in light gray; on $\operatorname{supp} a \backslash \operatorname{supp} e_{-}$, darker colors correspond to larger values of $a$. Also shown are the forward, resp. backward, trapped set $\Gamma_{-}$, resp. $\Gamma_{+}$, and the bicharacteristic flow nearby. The figure already suggests that $\mathrm{H}_{p}\left(a^{2}\right)$ is non-positive away from supp $e_{-}$, and actually negative away from $\operatorname{supp} e_{-} \cup \Gamma$; see equation $(2.3)$

lets $E_{-} \in \Psi_{h}(X)$ have principal symbol $e_{-}$, and wave front set in the support of $e_{-}$. This gives that

$$
\begin{aligned}
\frac{i}{4 h}\left[P, A^{*} A\right]= & -\left(C_{+} A_{+}\right)^{*}\left(C_{+} A_{+}\right)-\left(C_{-} A_{-}\right)^{*}\left(C_{-} A_{-}\right) \\
& +E_{-}^{*} E_{-}+h F
\end{aligned}
$$

for some $F \in \Psi_{h}(X)$ with

$$
\mathrm{WF}_{h}^{\prime}(F) \subset \operatorname{supp} a \text {. }
$$

Thus

$$
\frac{i}{4 h}\left\langle\left[P, A^{*} A\right] u, u\right\rangle=-\left\|C_{+} A_{+} u\right\|^{2}-\left\|C_{-} A_{-} u\right\|^{2}+\left\|E_{-} u\right\|^{2}+h\langle F u, u\rangle .
$$

Expanding the left hand side gives

$$
\begin{aligned}
& \left\langle P A^{*} A u, u\right\rangle-\left\langle A^{*} A P u, u\right\rangle \\
& \quad=\langle A u, A P u\rangle-\langle A P u, A u\rangle+\left\langle\left(P-P^{*}\right) A^{*} A u, u\right\rangle .
\end{aligned}
$$


As we are assuming that $P-P^{*}$ is $\mathcal{O}\left(h^{2}\right)$ near $\Gamma$, we may also assume that this holds on $\operatorname{supp} a$, thus the last term is $\mathcal{O}\left(h^{2}\right)\|u\|^{2}$. Thus,

$$
\left\|C_{+} A_{+} u\right\|^{2}+\left\|C_{-} A_{-} u\right\|^{2} \leq\left\|E_{-} u\right\|^{2}+h^{-1}|\langle A P u, A u\rangle|+C_{1} h\|u\|^{2}
$$

Now, by the duality of $\mathcal{H}_{h, \Gamma}$ and $\mathcal{H}_{h, \Gamma}^{*}$ relative to the $L^{2}$ inner product,

$$
\begin{aligned}
|\langle A P u, A u\rangle| & \leq\|A P u\|_{\mathcal{H}_{h, \Gamma}^{*}}\|A u\|_{\mathcal{H}_{h, \Gamma}} \\
& \leq \frac{h \epsilon}{2}\|A u\|_{\mathcal{H}_{h, \Gamma}}^{2}+\frac{1}{2 h \epsilon}\|A P u\|_{\mathcal{H}_{h, \Gamma}^{*}}^{2} .
\end{aligned}
$$

Next, for $\epsilon>0$ small, $\epsilon\left\|Q_{+} A u\right\|^{2}$ can be estimated in terms of $\left\|C_{+} A_{+} u\right\|^{2}+$ $\mathcal{O}(h)\|u\|^{2}$, as can be seen by comparing the principal symbols, in particular using the ellipticity of $C_{+}$on $\operatorname{supp} a$. One can thus absorb $\frac{\epsilon}{2}\|A u\|_{\mathcal{H}_{h, \Gamma}}^{2}$ into the left hand side of (2.5). This shows

$$
\left\|C_{+} A_{+} u\right\|^{2}+\left\|C_{-} A_{-} u\right\|^{2} \leq C\left\|E_{-} u\right\|^{2}+C h^{-2}\|A P u\|_{\mathcal{H}_{h, \Gamma}^{*}}^{2}+C h\|u\|^{2} .
$$

Now, since the region supp $e_{-}$is disjoint from $\Gamma_{+}$, it is backward nontrapped, and thus the standard propagation of singularities with complex absorption (see e.g., [3, Lemma 5.1]) implies that $E_{-} u$ is controlled by $P u$ microlocalized off $\Gamma_{+}$, hence by $Q_{+} P u$, modulo higher order (in $h$ ) terms in $P u$. This proves the first part of Theorem 1.1 since $A_{ \pm}$is an elliptic multiple of $Q_{ \pm}$microlocally near $\Gamma$. Thus, if we have a bound $\|u\| \leq C^{\prime} h^{-1-s}\|P u\|_{L^{2}}$, $0<s<1 / 2$, and thus $h\|u\|^{2} \leq C^{\prime} h^{-1-2 s}\|P u\|_{L^{2}}^{2} \leq C^{\prime \prime} h^{-1-2 s}\|P u\|_{\mathcal{H}_{h, \Gamma}^{*}}^{2}$, this implies a non-trapping estimate:

$$
\|u\|_{\mathcal{H}_{h, \Gamma}} \leq C h^{-1}\|P u\|_{\mathcal{H}_{h, \Gamma}^{*}}
$$

This completes the proof of Theorem 1.1.

In fact, as mentioned earlier, a slight change of point of view proves Theorem 1.1 directly. To see this, we use the Weyl quantization ${ }^{7}$ when choosing $a, a_{ \pm}, c_{ \pm}, e_{-}$; since we are on a manifold, this requires identifying functions with half-densities via trivialization of the half-density bundle by the Riemannian metric; this identification preserves self-adjointness. We also write $P_{h, z}$ as the Weyl quantization of $p_{0}+h p_{1}$ with $p_{0}, p_{1}$ real modulo

${ }^{7}$ The Weyl quantization is actually irrelevant. It is straightforward to see that if $A \in \Psi_{h}(X)$ and if the principal symbol of $A$ is real, then the real part of the subprincipal symbol is defined independently of choices. This is all that is needed for the argument below. 
$\mathcal{O}\left(h^{2}\right)$. Then the principal symbol calculation above holds with $p_{0}$ in place of $p$, and with $p_{1}$ included it yields additional terms

$$
\begin{aligned}
\frac{1}{4} \mathrm{H}_{p}\left(a^{2}\right)= & -\left(c_{+}^{2} \phi_{+}^{2}+c_{-}^{2} \phi_{-}^{2}-h \phi_{+} \mathrm{H}_{p_{1}} \phi_{+}+h \phi_{-} \mathrm{H}_{p_{1}} \phi_{-}\right) \\
& \times\left(\chi_{0} \chi_{0}^{\prime}\right)\left(\phi_{+}^{2}-\phi_{-}^{2}+\kappa\right) \chi\left(\phi_{+}^{2}\right)^{2} \psi(p)^{2} \\
& -\left(c_{+}^{2} \phi_{+}^{2}-h \phi_{+} \mathrm{H}_{p_{1}} \phi_{+}\right)\left(\chi^{\prime} \chi\right)\left(\phi_{+}^{2}\right) \chi_{0}\left(\phi_{+}^{2}-\phi_{-}^{2}+\kappa\right)^{2} \psi(p)^{2} .
\end{aligned}
$$

Now, (2.4) becomes

$$
\begin{aligned}
(2.7) \frac{i}{4 h}\left[P, A^{*} A\right]= & -\left(C_{+} A_{+}\right)^{*}\left(C_{+} A_{+}\right)-\left(C_{-} A_{-}\right)^{*}\left(C_{-} A_{-}\right) \\
& +h\left(A_{+}^{*} G_{+}+G_{+}^{*} A_{+}+A_{-}^{*} G_{-}+G_{-}^{*} A_{-}\right)+E+h^{2} F,
\end{aligned}
$$

with $G_{ \pm}$being the Weyl quantization of

$$
g_{ \pm}= \pm \frac{1}{2}\left(\mathrm{H}_{p_{1}} \phi_{ \pm}\right) \sqrt{\left(\chi_{0} \chi_{0}^{\prime}\right)\left(\phi_{+}^{2}-\phi_{-}^{2}+\kappa\right)} \chi\left(\phi_{+}^{2}\right) \psi(p)
$$

and with $F \in \Psi_{h}(X)$ with

$$
\mathrm{WF}_{h}^{\prime}(F) \subset \operatorname{supp} a \text {. }
$$

Correspondingly, (2.5) becomes

$$
\begin{aligned}
& \left\|C_{+} A_{+} u\right\|^{2}+\left\|C_{-} A_{-} u\right\|^{2} \\
& \quad \leq|\langle E u, u\rangle|+h^{-1}|\langle A P u, A u\rangle|+2 h\left\|A_{+} u\right\|\left\|G_{+} u\right\| \\
& \quad+2 h\left\|A_{-} u\right\|\left\|G_{-} u\right\|+C_{1} h^{2}\|u\|^{2} .
\end{aligned}
$$

The terms with $G_{ \pm}$on the right hand side can be estimated by

$$
\epsilon\left\|A_{+} u\right\|^{2}+\epsilon^{-1} h^{2}\left\|G_{+} u\right\|^{2}+\epsilon\left\|A_{-} u\right\|^{2}+\epsilon^{-1} h^{2}\left\|G_{-} u\right\|^{2},
$$

and for $\epsilon>0$ sufficiently small, the $\left\|A_{ \pm} u\right\|^{2}$ terms can now be absorbed into the left hand side of (2.8). Proceeding as above yields

$$
\begin{aligned}
\left\|C_{+} A_{+} u\right\|^{2}+\left\|C_{-} A_{-} u\right\|^{2} \leq & C|\langle E u, u\rangle|+C h^{-2}\|A P u\|_{\mathcal{H}_{h, \Gamma}^{*}}^{2} \\
& +C h^{2}\|u\|^{2} .
\end{aligned}
$$

Together with the non-trapping for the $E$ term this gives the global estimate

$$
\|u\|_{\mathcal{H}_{h, \Gamma}}^{2} \leq C h^{-2}\|P u\|_{\mathcal{H}_{h, \Gamma}^{*}}^{2}+C h^{2}\|u\|^{2},
$$


and now the last term on the right hand side can be absorbed into the left hand side for sufficiently small $h$, giving the estimate (1.5).

Notice that this also directly gives a weaker version of the WunschZworski estimate (1.2), namely

$$
\|u\|_{L^{2}} \leq C h^{-2}\|P u\|_{L^{2}}
$$

in view of the continuity of the inclusions $\mathcal{H}_{h, \Gamma} \hookrightarrow h^{-1 / 2} L^{2}$ and $h^{1 / 2} L^{2} \hookrightarrow$ $\mathcal{H}_{h, \Gamma}^{*}$

Remark 2.1. If one is interested in a fixed operator, rather than in a parameter-dependent family of operators, one can naturally strengthen the estimates $(1.4)-(1.5)$ by adding $h^{-1}\left\|\hat{P}_{0} u\right\|$ to the left-hand sides, where $\hat{P}_{0}$ is any elliptic multiple of $P$. A more natural way of phrasing such an improvement is to use "coisotropic, normally isotropic" spaces $\tilde{\mathcal{H}}_{h, \Gamma}$ and $\tilde{\mathcal{H}}_{h, \Gamma}^{*}$, where the squared norm on $\tilde{\mathcal{H}}_{h, \Gamma}$ is defined by

$$
\|u\|_{\tilde{\mathcal{H}}_{h, \Gamma}}^{2}=\left\|Q_{0} u\right\|^{2}+\left\|Q_{+} u\right\|^{2}+\left\|Q_{-} u\right\|^{2}+h^{-1}\left\|\hat{P}_{0} u\right\|^{2}+h\|u\|^{2},
$$

which strengthens the space and therefore weakens its dual. Using these spaces instead in (2.6), one obtains an additional term from $h\|A u\|_{\tilde{\mathcal{H}}_{h, \Gamma}}^{2}$, namely $\left\|\hat{P}_{0} A u\right\|^{2}$, which is bounded by $C\left(h^{-1}\|A P u\|_{\tilde{\mathcal{H}}_{h, \Gamma}^{*}}^{2}+h\|u\|^{2}\right)$, and thus the remainder of the second proof goes through.

\section{Non-trapping estimates in non-dilation invariant settings}

We now transfer Theorem 1.1 into the b-setting; the discussion in the previous section is essentially the dilation invariant special case of this, as we will explain below, although in the b-setting there is additional localization near the boundary. One main application of the b-estimate is in the analysis of linear and non-linear waves on asymptotically Kerr-de Sitter spaces; see $[6,10]$ for details.

\subsection{Notation and definitions}

For a general reference for b-analysis, see Melrose [7].

Let $M$ be an $n$-dimensional compact manifold with boundary $X$.

- Let $\mathcal{V}_{\mathrm{b}}(M)$ be the Lie algebra of b-vector fields on $M$, i.e., of vector fields on $M$ which are tangent to $X$. Elements of $\mathcal{V}_{\mathrm{b}}(M)$ are sections 


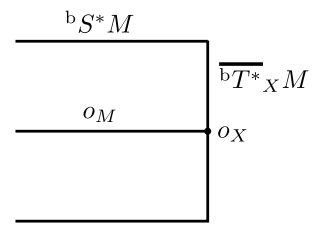

Figure 2: The radially compactified cotangent bundle $\overline{{ }^{\mathrm{b}} T^{*}} M$ near ${ }^{\overline{\mathrm{b}} T^{*}}{ }_{X} M$; the cosphere bundle ${ }^{\mathrm{b}} S^{*} M$, which is the boundary at fiber infinity of $\overline{\mathrm{b}} T^{*} M$, is also shown, as well as the zero section $o_{M} \subset \overline{\mathrm{b}} T^{*} M$ and the zero section over the boundary $o_{X} \subset \overline{\mathrm{b} T^{*}}{ }_{X} M$

of a natural vector bundle on $M$, namely the b-tangent bundle ${ }^{\mathrm{b}} T M$; in local coordinates $(\tau, x)$ near $X$, the fibers of ${ }^{\mathrm{b}} T M$ are spanned by $\tau \partial_{\tau}$ and $\partial_{x}$. The fibers of the dual bundle ${ }^{\mathrm{b}} T^{*} M$, called $b$-cotangent bundle, are spanned by $\frac{d \tau}{\tau}$ and $d x$.

It is often convenient to consider the fiber compactification $\overline{{ }^{\mathrm{b}} T^{*}} M$ of ${ }^{\mathrm{b}} T^{*} M$, where the fibers are replaced by their radial compactification. The new boundary of $\overline{\mathrm{b}} T^{*} M$ at fiber infinity is the $b$-cosphere bundle ${ }^{\mathrm{b}} S^{*} M$; it still possesses the compactification of the "old" boundary $\overline{{ }^{\mathrm{b}} T^{*}} X M$, see Figure $2 .{ }^{\mathrm{b}} S^{*} M$ is naturally the quotient of ${ }^{\mathrm{b}} T^{*} M \backslash o$ by the $\mathbb{R}^{+}$-action of dilation in the fibers of the cotangent bundle. Many sets that we will consider below are conic subsets of ${ }^{\mathrm{b}} T^{*} M \backslash o$, and we will often view them as subsets of ${ }^{\mathrm{b}} S^{*} M$.

- For $a \in \mathcal{C}^{\infty}\left({ }^{\mathrm{b}} T^{*} M\right)$, we say $a \in S^{m}\left({ }^{\mathrm{b}} T^{*} M\right)$ if $a$ satisfies

$$
\left|\partial_{z}^{\alpha} \partial_{\zeta}^{\beta} a(z, \zeta)\right| \leq C_{\alpha \beta}\langle\zeta\rangle^{m-|\beta|} \text { for all multiindices } \alpha, \beta
$$

in any coordinate chart, where $z$ are coordinates in the base and $\zeta$ coordinates in the fiber; more precisely, in local coordinates $(\tau, x)$ near $X$, we take $\zeta=(\sigma, \xi)$, where we write b-covectors as

$$
\sigma \frac{d \tau}{\tau}+\sum_{j} \xi_{j} d x_{j}
$$

We define the quantization $\operatorname{Op}(a)$ of $a$, acting on smooth functions $u$ supported in a coordinate chart, by

$$
\begin{array}{r}
\operatorname{Op}(a) u(\tau, x)=(2 \pi)^{-n} \int e^{i\left(\tau-\tau^{\prime}\right) \tilde{\sigma}+i\left(x-x^{\prime}\right) \xi} \phi\left(\frac{\tau-\tau^{\prime}}{\tau}\right) \\
\quad \times a(\tau, x, \tau \tilde{\sigma}, \xi) u\left(\tau^{\prime}, x^{\prime}\right) d \tau^{\prime} d x^{\prime} d \tilde{\sigma} d \xi,
\end{array}
$$


where the $\tau^{\prime}$-integral is over $[0, \infty)$, and $\phi \in \mathcal{C}_{\mathrm{c}}^{\infty}((-1 / 2,1 / 2))$ is identically 1 near $0 .{ }^{8}$ For general $u$, define $\mathrm{Op}(a) u$ using a partition of unity. We write $\operatorname{Op}(a) \in \Psi_{\mathrm{b}}^{m}(M)$. We say that $a$ is a symbol of $\operatorname{Op}(a)$. The equivalence class of $a$ in $S^{m}\left({ }^{\mathrm{b}} T^{*} M\right) / S^{m-1}\left({ }^{\mathrm{b}} T^{*} M\right)$ is invariantly defined on ${ }^{\mathrm{b}} T^{*} M$ and is called the principal symbol of $\mathrm{Op}(a)$. We will tacitly assume that all our operators have homogeneous principal symbols.

- If $A \in \Psi_{\mathrm{b}}^{m_{1}}(M)$ and $B \in \Psi_{\mathrm{b}}^{m_{2}}(M)$, then $[A, B] \in \Psi_{\mathrm{b}}^{m_{1}+m_{2}-1}(M)$, and its principal symbol is $\frac{1}{i} \mathrm{H}_{a} b \equiv \frac{1}{i}\{a, b\}$, where the Hamilton vector field $\mathrm{H}_{a}$ of the principal symbol $a$ of $A$ is the extension of the Hamilton vector field from $T^{*} M^{\circ} \backslash o$ to ${ }^{\mathrm{b}} T^{*} M \backslash o$, which is a homogeneous degree $m-1$ vector field on ${ }^{\mathrm{b}} T^{*} M \backslash o$ tangent to the boundary ${ }^{\mathrm{b}} T_{X}^{*} M$. In local coordinates $(\tau, x, \sigma, \xi)$ on ${ }^{\mathrm{b}} T^{*} M$ as above, this has the form

$$
\mathrm{H}_{a}=\left(\partial_{\sigma} a\right)\left(\tau \partial_{\tau}\right)-\left(\tau \partial_{\tau} a\right) \partial_{\sigma}+\sum_{j}\left(\left(\partial_{\xi_{j}} a\right) \partial_{x_{j}}-\left(\partial_{x_{j}} a\right) \partial_{\xi_{j}}\right)
$$

- We define bicharacteristics completely analogously to the semiclassical setting.

- The microsupport $\mathrm{WF}_{\mathrm{b}}^{\prime}(A) \subset{ }^{\mathrm{b}} T^{*} M \backslash o$ of $A=\mathrm{Op}(a) \in \Psi_{\mathrm{b}}^{m}(M)$ is the complement of the set of all $\rho \in{ }^{\mathrm{b}} T^{*} M \backslash o$ such that $a$ is rapidly decaying in a conic neighborhood around $\rho$. Note that $\mathrm{WF}_{\mathrm{b}}^{\prime}(A)$ is conic, hence we will also view it as a subset of ${ }^{\mathrm{b}} S^{*} M$.

- Fix a $b$-density on $M$, which is locally of the form $a\left|\frac{d \tau}{\tau} d z\right|, a>0$.

- Define the $b$-Sobolev space $H_{\mathrm{b}}^{k}(M)$ for $k \in \mathbb{Z}_{\geq 0}$ by

$$
H_{\mathrm{b}}^{k}(M)=\left\{u \in L^{2}(M): X_{1} \cdots X_{k} u \in L^{2}(M), X_{1}, \ldots, X_{j} \in \mathcal{V}_{\mathrm{b}}(M)\right\}
$$

and for general $k \in \mathbb{R}$ by duality and interpolation. Moreover, define the weighted b-Sobolev spaces $H_{\mathrm{b}}^{s, \alpha}(M):=\tau^{\alpha} H_{\mathrm{b}}^{s}(M)$ for $s, \alpha \in \mathbb{R}$, where $\tau$ is a boundary defining function, i.e., $\tau=0$ at $X$ and $d \tau \neq 0$ there. Every b-pseudodifferential operator $A \in \Psi_{\mathrm{b}}^{m}(M)$ is a bounded operator $A: H_{\mathrm{b}}^{s, \alpha}(M) \rightarrow H_{\mathrm{b}}^{s-m, \alpha}(M), s, \alpha \in \mathbb{R}$.

\footnotetext{
${ }^{8}$ The cutoff $\phi$ ensures that these operators lie in the "small b-calculus" of Melrose, in particular that such quantizations act on weighted b-Sobolev spaces, defined below.
} 
- For $A \in \Psi_{\mathrm{b}}^{m}(M)$ with principal symbol $a \in S^{m}\left({ }^{\mathrm{b}} T^{*} M\right)$, we say that $A$ is elliptic at $\rho \in{ }^{\mathrm{b}} T^{*} M \backslash o$ if there is a constant $C>0$ such that $|a(z, \zeta)| \geq C|\zeta|^{m}$ for $(z, \zeta)$ in a conic neighborhood of $\rho$. The characteristic set of $A$ is the complement (in ${ }^{\mathrm{b}} T^{*} M \backslash o$ ) of the set of all $\rho$ at which $A$ is elliptic.

- For $u \in H_{\mathrm{b}}^{-\infty, \alpha}(M)$, define its $H_{\mathrm{b}}^{s, \alpha}$ wave front set $\mathrm{WF}_{\mathrm{b}}^{s, \alpha}(u) \subset{ }^{\mathrm{b}} T^{*} M \backslash$ $o$ as the complement of the set of all $\rho \in{ }^{\mathrm{b}} T^{*} M \backslash o$ for which there exists $a \in S^{0}\left({ }^{\mathrm{b}} T^{*} M\right)$ elliptic at $\rho$ such that $\mathrm{Op}(a) u \in H_{\mathrm{b}}^{s, \alpha}(M)$. In particular, $\mathrm{WF}_{\mathrm{b}}^{s, \alpha}(u)=\emptyset$ if and only if $u \in H_{\mathrm{b}}^{s, \alpha}(M)$.

- Microlocal elliptic regularity states that if $A u=f$ with $A \in \Psi_{\mathrm{b}}^{m}(M)$, $u, f \in H_{\mathrm{b}}^{-\infty, \alpha}(M), \rho \notin \mathrm{WF}_{\mathrm{b}}^{s-m, \alpha}(f)$ and $A$ is elliptic at $\rho$, then $\rho \notin$ $\mathrm{WF}_{\mathrm{b}}^{s, \alpha}(u)$.

- If $A \in \Psi_{\mathrm{b}}^{m}(M)$ has a principal symbol with non-positive imaginary part, $u, f \in H_{\mathrm{b}}^{-\infty, \alpha}(M), \quad A u=f$, moreover $\rho \notin \mathrm{WF}_{\mathrm{b}}^{s, \alpha}(u)$ and $\gamma_{\rho}([0, T]) \cap \mathrm{WF}_{\mathrm{b}}^{s-m+1, \alpha}(f)=\emptyset$ for some $T>0$, then the propagation of singularities states that $\gamma_{\rho}(T) \notin \mathrm{WF}_{\mathrm{b}}^{s, \alpha}(u)$.

\subsection{Setup, statement and proof of the result}

Suppose $\mathcal{P} \in \Psi_{\mathrm{b}}^{m}(M), \mathcal{P}-\mathcal{P}^{*} \in \Psi_{\mathrm{b}}^{m-2}(M)$. Let $p$ be the principal symbol of $\mathcal{P}$, which is thus a homogeneous degree $m$ function on ${ }^{\mathrm{b}} T^{*} M \backslash o$, which we assume to be real-valued. Let $\tilde{\rho}$ denote a homogeneous degree -1 defining function of ${ }^{\mathrm{b}} S^{*} M$. Then the rescaled Hamilton vector field

$$
V=\tilde{\rho}^{m-1} \mathrm{H}_{p}
$$

is a $\mathcal{C}^{\infty}$ vector field on $\overline{\mathrm{b}} T^{*} M$ away from the 0 -section, and it is tangent to all boundary faces. The characteristic set $\Sigma$ is the zero-set of the smooth function $\tilde{\rho}^{m} p$ in ${ }^{\mathrm{b}} S^{*} M$. We will, somewhat imprecisely, refer to the flow of $V$ in $\Sigma \subset{ }^{\mathrm{b}} S^{*} M$ as the Hamilton, or (null)bicharacteristic flow; its integral curves, the (null)bicharacteristics, are reparameterizations of those of the Hamilton vector field $\mathrm{H}_{p}$, projected by the quotient map ${ }^{\mathrm{b}} T^{*} M \backslash o \rightarrow{ }^{\mathrm{b}} S^{*} M$.

We first work microlocally near the trapped set, namely assume that

(1) $\Gamma \subset \Sigma \cap{ }^{\mathrm{b}} S_{X}^{*} M$ is a smooth submanifold disjoint from the image of $T^{*} X \backslash o$ (so $\tau D_{\tau}$ is elliptic near $\Gamma$ ),

(2) $\Gamma_{+}$is a smooth submanifold of $\Sigma \cap{ }^{\mathrm{b}} S_{X}^{*} M$ in a neighborhood $U_{1}$ of $\Gamma$,

(3) $\Gamma_{-}$is a smooth submanifold of $\Sigma$ transversal to $\Sigma \cap{ }^{\mathrm{b}} S_{X}^{*} M$ in $U_{1}$, 


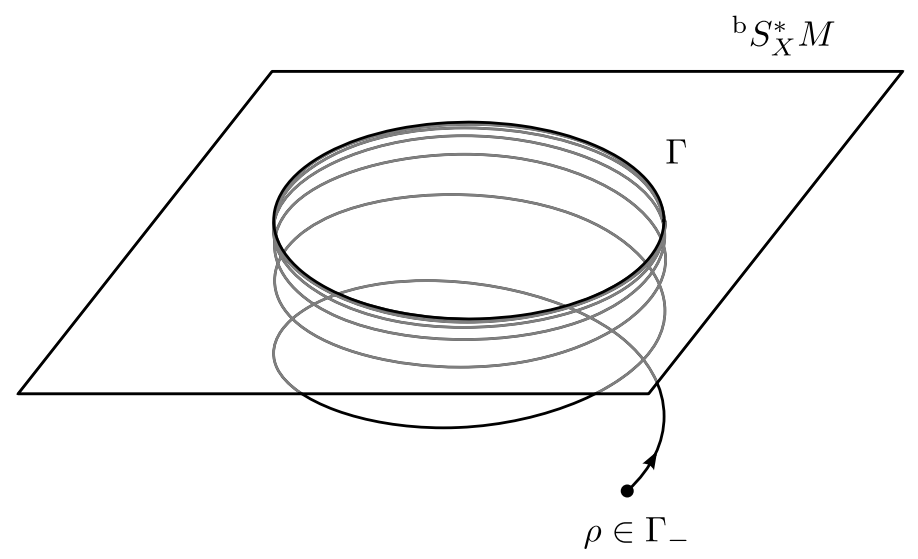

Figure 3: An exemplary situation with trapping: Shown are the (projection from ${ }^{\mathrm{b}} S^{*} M$ to the base $M$ of the) trapped set $\Gamma$, the b-cosphere bundle over $X$ as well as a forward bicharacteristic starting at a point $\rho \in \Gamma_{-}$

(4) $\Gamma_{+}$has codimension 2 in $\Sigma, \Gamma_{-}$has codimension 1 ,

(5) $\Gamma_{+}$and $\Gamma_{-}$intersect transversally in $\Sigma$ with $\Gamma_{+} \cap \Gamma_{-}=\Gamma$,

(6) the rescaled Hamilton vector field $V=\tilde{\rho}^{m-1} \mathrm{H}_{p}$ is tangent to both $\Gamma_{+}$ and $\Gamma_{-}$, and thus to $\Gamma$.

We assume that $\Gamma_{+}$is backward trapped for the Hamilton flow (i.e., bicharacteristics in $\Gamma_{+}$near $\Gamma$ tend to $\Gamma$ as the parameter goes to $-\infty$ ), i.e., is the unstable manifold of $\Gamma$, while $\Gamma_{-}$is forward trapped, i.e., is the stable manifold of $\Gamma$, see Figure 3 ; indeed, we assume a quantitative version of this. (There is a completely analogous statement if $\Gamma_{+}$is forward trapped and $\Gamma_{-}$is backward trapped: replacing $\mathcal{P}$ by $-\mathcal{P}$ preserves all assumptions, but reverses the Hamilton flow.) To state this, let $\phi_{-}$be a defining function of $\Gamma_{-}$, and let $\phi_{+} \in \mathcal{C}^{\infty}\left({ }^{\mathrm{b}} S^{*} M\right)$ be a defining function of $\Gamma_{+}$in ${ }^{\mathrm{b}} S_{X}^{*} M$; thus $\Gamma_{+}$is defined within ${ }^{\mathrm{b}} S^{*} M$ by $\tau=0, \phi_{+}=0$. Notice that $V$ being to tangent to ${ }^{\mathrm{b}} S_{X}^{*} M$ (due to (3.1)) implies that $V \tau$ is a multiple of $\tau$; we assume that, near $\Gamma$,

$$
V \tau=-c_{\partial}^{2} \tau, c_{\partial}>0
$$

this is consistent with the stability of $\Gamma_{-}$. By the tangency requirement, with

$$
\hat{p}_{0}=\tilde{\rho}^{m} p
$$


$V \phi_{-}=\alpha_{-} \phi_{-}+\nu_{-} \hat{p}_{0}, \alpha_{-}$smooth; notice that changing $\phi_{-}$by a smooth nonzero multiple $f$ gives $V\left(f \phi_{-}\right)=\alpha_{-} f \phi_{-}+\nu_{-} f \hat{p}_{0}+(V f) \phi_{-}$, so $\alpha_{-}$depends on the choice of $\phi_{-}$. On the other hand, the tangency requirement gives $V \phi_{+}=\alpha_{+} \phi_{+}+\beta_{+} \tau+\nu_{+} \hat{p}_{0}$. For the sake of conciseness, rather than stating the assumptions on the Hamilton flow as in [12], we assume directly that $\phi_{ \pm}$satisfy

$$
V \phi_{-}=c_{-}^{2} \phi_{-}+\nu_{-} \hat{p}_{0}, V \phi_{+}=-c_{+}^{2} \phi_{+}+\beta_{+} \tau+\nu_{+} \hat{p}_{0},
$$

with $c_{ \pm}>0$ smooth near $\Gamma, \beta_{+}, \nu_{ \pm}$smooth near $\Gamma$ and

$$
\left\{\phi_{+}, \phi_{-}\right\}>0
$$

near $\Gamma$. Let $U_{0} \subset \overline{U_{0}} \subset U_{1}$ be a neighborhood of $\Gamma$ such that the Poisson bracket in (3.4) as well as $c_{ \pm}$have positive lower bounds.

Now, given a boundary defining function $\tau,{ }^{\mathrm{b}} S_{X}^{*} M \backslash S^{*} X$ (where $S^{*} X$ is the image of $T^{*} X$ under the $\mathbb{R}^{+}$-dilation quotient) can be identified with $\left(\frac{d \tau}{\tau}+T^{*} X\right) \cup\left(-\frac{d \tau}{\tau}+T^{*} X\right)$ (which in turn can be identified with two copies of $\left.T^{*} X\right)$ since ${ }^{\mathrm{b}} T_{X}^{*} M=\operatorname{Span}\left\{\frac{d \tau}{\tau}\right\} \oplus T^{*} X$, and each $\mathbb{R}^{+}$-orbit outside $T^{*} X$ intersects $\left(\frac{d \tau}{\tau}+T^{*} X\right) \cup\left(-\frac{d \tau}{\tau}+T^{*} X\right)$ in a unique point. This provides the connection between the b- and the semiclassical perspectives, i.e., analysis on ${ }^{\mathrm{b}} T_{X}^{*} M$ and that of $T^{*} X$. In fact, if $p$ is a homogeneous degree $m$ function, then $\tilde{\rho}^{m} p$, where $\tilde{\rho}$ can be taken as the reciprocal of the absolute value of the symbol of $\tau D_{\tau}$ in this region (which is well-defined, independent of choices), gives a function on $\{ \pm 1\} \times T^{*} X$; this is exactly the semiclassical rescaling, with $\tilde{\rho}^{m} p$ the semiclassical principal symbol (depending on the parameter \pm 1 ) of the rescaled operator family (cf. [10, Section 2.1]).

Notice that if we merely assume the normal hyperbolicity within ${ }^{\mathrm{b}} S_{X}^{*} M$, in the sense of this identification with $T^{*} X$, as in [12, Section 1.2], then [12, Lemma 4.1], as corrected in [11], actually gives such defining functions $\phi_{ \pm}^{0}$ within ${ }^{\mathrm{b}} S_{X}^{*} M$ (i.e., letting $\tau=0$ ); taking an arbitrary extension in case of $\phi_{+}$, and an extension which is a defining function in case of $\Gamma_{-}$, all the requirements above are satisfied. In particular, Kerr and Kerr-de Sitter spaces satisfy these assumptions, as do their perturbations when the angular momentum $|a|$ is small; see [12, Proposition 2.1] for the Kerr setting and $[10$, Section 6] for the Kerr-de Sitter one. Indeed, in the Kerr case the full range of $|a|<M, M$ the black hole mass, satisfies the hypotheses, as shown by Dyatlov [4]. 
There is an asymmetry between the roles of $\phi_{ \pm}$and $\tau$, and thus we consider the parabolic defining function

$$
\rho_{+}=\phi_{+}^{2}+\mathrm{M} \tau
$$

for $\Gamma_{+}, M>0$, to be chosen. Then, near $\Gamma$,

$$
\begin{aligned}
\hat{\rho}_{+}=V \rho_{+} & =-2 c_{+}^{2} \phi_{+}^{2}+2 \beta_{+} \phi_{+} \tau+2 \nu_{+} \phi_{+} \hat{p}_{0}-\mathrm{M} c_{\partial}^{2} \tau \\
& =-2 c_{+}^{2} \phi_{+}^{2}-\left(\mathrm{M} c_{\partial}^{2}-2 \beta_{+} \phi_{+}\right) \tau+2 \nu_{+} \phi_{+} \hat{p}_{0} \\
& \leq-\tilde{c}_{+}^{2} \rho_{+}+2 \nu_{+} \phi_{+} \hat{p}_{0}, \quad \tilde{c}_{+}>0,
\end{aligned}
$$

if $M>0$ is chosen sufficiently large, consistently with the forward trapped nature of $\Gamma_{-}$. (Here the term with $\hat{p}_{0}$ is considered harmless as one essentially restricts to the characteristic set, $\hat{p}_{0}=0$.) Also, note that one can use ${ }^{9}$ the reciprocal $\tilde{\rho}=|\sigma|^{-1}$ of the principal symbol $\sigma$ of $\tau D_{\tau}$ as the local defining function of ${ }^{\mathrm{b}} S^{*} M$ as fiber-infinity in ${ }^{\mathrm{b}} T^{*} M$ near $\Gamma$; then

$$
V \tilde{\rho}=\tilde{\alpha} \tilde{\rho} \tau
$$

for some $\tilde{\alpha}$ smooth in view of (3.1).

Similar to the normally isotropic spaces in the semiclassical setting, we introduce spaces which are normally isotropic at $\Gamma{ }^{10}$ Concretely, let $Q_{ \pm} \in \Psi_{\mathrm{b}}^{0}(M)$ have principal symbol $\phi_{ \pm}$as before, $\hat{P}_{0} \in \Psi_{\mathrm{b}}^{0}(M)$ have principal symbol $\hat{p}_{0}$ and let $Q_{0} \in \Psi_{\mathrm{b}}^{0}(M)$ be elliptic, with real principal symbol for convenience, on $U_{0}^{c}$ (and thus nearby). Define the (global) b-normally isotropic spaces at $\Gamma$ of order $s, \mathcal{H}_{\mathrm{b}, \Gamma}^{s}$, by the norm

$$
\begin{aligned}
\|u\|_{\mathcal{H}_{\mathrm{b}, \Gamma}^{s}}^{2}= & \left\|Q_{0} u\right\|_{H_{\mathrm{b}}^{s}}^{2}+\left\|Q_{+} u\right\|_{H_{\mathrm{b}}^{s}}^{2}+\left\|Q_{-} u\right\|_{H_{\mathrm{b}}^{s}}^{2}+\left\|\tau^{1 / 2} u\right\|_{H_{\mathrm{b}}^{s}}^{2} \\
& +\left\|\hat{P}_{0} u\right\|_{H_{\mathrm{b}}^{s}}^{2}+\|u\|_{H_{\mathrm{b}}^{s-1 / 2}}^{2}
\end{aligned}
$$

\footnotetext{
${ }^{9}$ Indeed, in the semiclassical setting, after Mellin transforming this problem, $|\sigma|^{-1}$ plays the role of the semiclassical parameter $h$, which in that case commutes with the operator.

${ }^{10}$ Note that ${ }^{\mathrm{b}} T^{*} M$ is not a symplectic manifold (in a natural way) since the symplectic form on ${ }^{\mathrm{b}} T_{M}^{*}{ }^{\circ} M$ does not extend smoothly to ${ }^{\mathrm{b}} T^{*} M$. Thus, the word "normally isotropic" is not completely justified; we use it since it reflects that in the analogous semiclassical setting, see [12], the set $\Gamma$ is symplectic, and the origin in the symplectic orthocomplement $\left(T_{\alpha} \Gamma\right)^{\perp}$ of $T_{\alpha} \Gamma$, which is also symplectic, is isotropic within $\left(T_{\alpha} \Gamma\right)^{\perp}$.
} 
and let $\mathcal{H}_{\mathrm{b}, \Gamma}^{*,-s}$ be the dual space relative to $L^{2}$, which is thus ${ }^{11}$

$$
Q_{0} H_{\mathrm{b}}^{-s}+Q_{+} H_{\mathrm{b}}^{-s}+Q_{-} H_{\mathrm{b}}^{-s}+\tau^{1 / 2} H_{\mathrm{b}}^{-s}+\hat{P}_{0} H_{\mathrm{b}}^{-s}+H_{\mathrm{b}}^{-s+1 / 2} .
$$

Note that microlocally away from $\Gamma, \mathcal{H}_{\mathrm{b}, \Gamma}^{s}$ is just the standard $H_{\mathrm{b}}^{s}$ space, while $\mathcal{H}_{\mathrm{b}, \Gamma}^{*,-s}$ is $H_{\mathrm{b}}^{-s}$ since at least one of $Q_{0}, Q_{ \pm}$and $\tau$ is elliptic. Moreover, $\Psi_{\mathrm{b}}^{k}(M) \ni A: \mathcal{H}_{\mathrm{b}, \Gamma}^{s} \rightarrow \mathcal{H}_{\mathrm{b}, \Gamma}^{s-k}$ is continuous since $\left[Q_{+}, A\right] \in \Psi_{\mathrm{b}}^{k-1}(M)$ etc.; the analogous statement also holds for the dual spaces. Further, the last term in (3.7) can be replaced by $\|u\|_{H_{\mathrm{b}}^{s-1}}^{2}$ as $i\left[Q_{+}, Q_{-}\right]=B^{*} B+R, B \in \Psi_{\mathrm{b}}^{-1 / 2}(M)$, $R \in \Psi_{\mathrm{b}}^{-2}(M)$, using the same argument as in the semiclassical setting (however, it cannot be dropped altogether unlike in the semiclassical setting!).

Remark 3.1. The notation $\mathcal{H}_{\mathrm{b}, \Gamma}^{s}(M)$ is justified for the space is independent of the particular defining functions $\phi_{ \pm}$chosen; near $\Gamma$ any other choice would replace $\phi_{ \pm}$by smooth non-degenerate linear combinations plus a multiple of $\tau$ and of $\hat{p}_{0}$, denote these by $\tilde{\phi}_{ \pm}$, and thus the corresponding $\tilde{Q}_{ \pm}$can be expressed as

$$
\begin{aligned}
& B_{+} Q_{+}+B_{-} Q_{-}+B_{\partial} \tau+\hat{B} \hat{P}_{0}+B_{0} Q_{0}+R, \\
& B_{ \pm}, B_{0}, B_{\partial}, \hat{B} \in \Psi_{\mathrm{b}}^{0}(M), \quad R \in \Psi_{\mathrm{b}}^{-1}(M),
\end{aligned}
$$

so the new norm can be controlled by the old norm, and conversely in view of the non-degeneracy.

Our result is then:

Theorem 3.2. With $\mathcal{P}, \mathcal{H}_{\mathrm{b}, \Gamma}^{s}, \mathcal{H}_{\mathrm{b}, \Gamma}^{*, s}$ as above, for any neighborhood $U$ of $\Gamma$ and for any $N$ there exist $B_{0} \in \Psi_{\mathrm{b}}^{0}(M)$ elliptic at $\Gamma$ and $B_{1}, B_{2} \in \Psi_{\mathrm{b}}^{0}(M)$ with $\mathrm{WF}_{\mathrm{b}}^{\prime}\left(B_{j}\right) \subset U, j=0,1,2, \mathrm{WF}_{\mathrm{b}}^{\prime}\left(B_{2}\right) \cap \Gamma_{+}=\emptyset$ and $C>0$ such that

$$
\left\|B_{0} u\right\|_{\mathcal{H}_{\mathrm{b}, \Gamma}^{s}} \leq\left\|B_{1} \mathcal{P} u\right\|_{\mathcal{H}_{\mathrm{b}, \Gamma}^{*, s-m+1}}+\left\|B_{2} u\right\|_{H_{\mathrm{b}}^{s}}+C\|u\|_{H_{\mathrm{b}}^{-N}}
$$

i.e., if all the functions on the right hand side are in the indicated spaces: $B_{1} \mathcal{P} u \in \mathcal{H}_{\mathrm{b}, \Gamma}^{*, s-m+1}$, etc., then $B_{0} u \in \mathcal{H}_{\mathrm{b}, \Gamma}^{s}$, and the inequality holds.

${ }^{11}$ We refer to $[8$, Appendix A] for a general discussion of the underlying functional analysis. In particular, Lemma A.3 there essentially gives the density of $\dot{\mathcal{C}}^{\infty}(M)$ in $\mathcal{H}_{\mathrm{b}, \Gamma}^{s}(M)$ : one can simply drop the subscript "e" in the statement of that lemma to conclude that $H_{\mathrm{b}}^{\infty}(M)$ (so in particular $H_{\mathrm{b}}^{s}(M)$ ) is dense in $\mathcal{H}_{\mathrm{b}, \Gamma}^{s}(M)$, and then the density of $\dot{\mathcal{C}}^{\infty}(M)$ in $H_{\mathrm{b}}^{s^{\prime}}(M)$ for any $s^{\prime}$ completes the argument. The completeness of $\mathcal{H}_{\mathrm{b}, \Gamma}^{s}(M)$ follows from the continuity of $\Psi_{\mathrm{b}}^{0}(M)$ on $H_{\mathrm{b}}^{s-1 / 2}(M)$. 
The same conclusion also holds if we assume $\mathrm{WF}_{\mathrm{b}}^{\prime}\left(B_{2}\right) \cap \Gamma_{-}=\emptyset$ instead of $\mathrm{WF}_{\mathrm{b}}^{\prime}\left(B_{2}\right) \cap \Gamma_{+}=\emptyset$.

Finally, if $r<0$, then, with $\mathrm{WF}_{\mathrm{b}}^{\prime}\left(B_{2}\right) \cap \Gamma_{+}=\emptyset$, (3.8) becomes

$$
\left\|B_{0} u\right\|_{H_{\mathrm{b}}^{s, r}} \leq\left\|B_{1} \mathcal{P} u\right\|_{H_{\mathrm{b}}^{s-m+1, r}}+\left\|B_{2} u\right\|_{H_{\mathrm{b}}^{s, r}}+C\|u\|_{H_{\mathrm{b}}^{-N, r}},
$$

while if $r>0$, then, with $\mathrm{WF}_{\mathrm{b}}^{\prime}\left(B_{2}\right) \cap \Gamma_{-}=\emptyset$,

$$
\left\|B_{0} u\right\|_{H_{\mathrm{b}}^{s, r}} \leq\left\|B_{1} \mathcal{P} u\right\|_{H_{\mathrm{b}}^{s-m+1, r}}+\left\|B_{2} u\right\|_{H_{\mathrm{b}}^{s, r}}+C\|u\|_{H_{\mathrm{b}}^{-N, r}},
$$

Remark 3.3. Note that the weighted versions (3.9) and (3.10) use standard weighted b-Sobolev spaces; this corresponds to non-trapping semiclassical estimates if the subprincipal symbol has the correct, definite, sign at $\Gamma$.

Proof. We may assume that $U \subset U_{0}$ is disjoint from a neighborhood of $\mathrm{WF}_{\mathrm{b}}^{\prime}\left(Q_{0}\right)$, and thus ignore $Q_{0}$ in the definition of $\mathcal{H}_{\mathrm{b}, \Gamma}^{s}$ below.

The main part of the proof is to show that there exist $B_{0}, B_{1}, B_{2}$ as above and $B_{3} \in \Psi_{\mathrm{b}}^{0}(M)$ with $\mathrm{WF}_{\mathrm{b}}^{\prime}\left(B_{3}\right) \subset U$ such that

$$
\left\|B_{0} u\right\|_{\mathcal{H}_{\mathrm{b}, \Gamma}^{s}} \leq\left\|B_{1} \mathcal{P} u\right\|_{\mathcal{H}_{\mathrm{b}, \Gamma}^{*, s-m+1}}+\left\|B_{2} u\right\|_{H_{\mathrm{b}}^{s}}+\left\|B_{3} u\right\|_{H_{\mathrm{b}}^{s-1}}+C\|u\|_{H_{\mathrm{b}}^{-N}}
$$

An iterative argument will then prove the theorem.

The proof is a straightforward modification of the construction in the semiclassical setting above, replacing $\phi_{+}^{2}$ by $\phi_{+}^{2}+\mathrm{M} \tau, \mathrm{M}>0$ large, in accordance with (3.5).

We start by pointing out that for any $\tilde{B}_{0} \in \Psi_{\mathrm{b}}^{0}(M)$ and any $\tilde{B}_{3} \in \Psi_{\mathrm{b}}^{0}(M)$ elliptic on $\mathrm{WF}_{\mathrm{b}}^{\prime}\left(\tilde{B}_{0}\right)$, we have

$$
\left\|\hat{P}_{0} \tilde{B}_{0} u\right\|_{H_{\mathrm{b}}^{s}} \leq C\left\|\tilde{B}_{0} \mathcal{P} u\right\|_{H_{\mathrm{b}}^{s-m}}+C^{\prime}\left\|\tilde{B}_{3} u\right\|_{H_{\mathrm{b}}^{s-1}}
$$

by using that $\hat{P}_{0}$ is an elliptic multiple of $\mathcal{P}$ modulo $\Psi_{\mathrm{b}}^{-1}(M)$. Since $\left\|\tilde{B}_{0} \mathcal{P} u\right\|_{H_{\mathrm{b}}^{s-m}} \leq C\left\|\tilde{B}_{0} \mathcal{P} u\right\|_{\mathcal{H}_{\mathrm{b},}^{*, s-m}}$, the $\hat{P}_{0}$ contribution to $\left\|\tilde{B}_{0} u\right\|_{\mathcal{H}_{\mathrm{b}, \Gamma}^{s}}$ in $(3.11)$ is thus automatically controlled.

So let $\chi_{0}(t)=e^{-\gamma / t}$ for $t>0, \chi_{0}(t)=0$ for $t \leq 0$, with $\gamma>0$ (large) to be specified, $\chi \in \mathcal{C}_{c}^{\infty}([0, \infty))$ be identically 1 near 0 with $\chi^{\prime} \leq 0$, and indeed with $\chi^{\prime} \chi=-\chi_{1}^{2}, \chi_{1} \geq 0, \chi_{1} \in \mathcal{C}_{c}^{\infty}([0, \infty))$, and let $\psi \in \mathcal{C}_{c}^{\infty}(\mathbb{R})$ be identically 1 near 0 . As we use the Weyl quantization, ${ }^{12}$ we write $\mathcal{P}$ as

\footnotetext{
${ }^{12}$ Again, the Weyl quantization is irrelevant: if $A \in \Psi_{\mathrm{b}}^{m}(X)$ and the principal symbol of $A$ is real, then the real part of the subprincipal symbol is defined independently of choices, which suffices below.
} 
the Weyl quantization of $p=p_{0}+\tilde{\rho} p_{1}$, with $\tilde{\rho} p_{1}$ of order $m-1$. Let

$$
a=\tilde{\rho}^{-s+(m-1) / 2} \chi_{0}\left(\rho_{+}-\phi_{-}^{2}+\kappa\right) \chi\left(\rho_{+}\right) \psi\left(\tilde{\rho}^{m} p\right)
$$

$\kappa>0$ small. Notice that on $\operatorname{supp} a$, if $\chi$ is supported in $[0, R]$,

$$
\rho_{+} \leq R, \phi_{-}^{2} \leq \rho_{+}+\kappa=R+\kappa
$$

so $a$ is localized near $\Gamma$ if $R$ and $\kappa$ are taken sufficiently small. In particular, the argument of $\chi_{0}$ is bounded above by $R+\kappa$, so given any $\mathrm{M}_{0}>0$ one can take $\gamma>0$ large so that

$$
\chi_{0}^{\prime} \chi_{0}-\mathrm{M}_{0} \chi_{0}^{2}=b^{2} \chi_{0}^{\prime} \chi_{0},
$$

with $b \geq 1 / 2, \mathcal{C}^{\infty}$, on the range of the argument of $\chi_{0}$.

In fact, we also need to regularize, namely introduce

$$
a_{\epsilon}=\left(1+\epsilon \tilde{\rho}^{-1}\right)^{-2} a, \epsilon \in[0,1]
$$

which is a symbol of order $s-(m-1) / 2-2$ for $\epsilon>0$, and is uniformly bounded in symbols of order $s-(m-1) / 2$ as $\epsilon$ varies in $[0,1]$. In order to avoid more cumbersome notation below, we ignore the regularizer and work directly with $a$; since the regularizer gives the same kind of contributions to the commutator as the weight $\tilde{\rho}^{-s+(m-1) / 2}$, these contributions can be dominated in exactly the same way.

Then, with $p=p_{0}+\tilde{\rho} p_{1}$ as above, $W=\tilde{\rho}^{m-2} \mathrm{H}_{\tilde{\rho} p_{1}}$, which is a smooth vector field near ${ }^{\mathrm{b}} S^{*} M$ as $\tilde{\rho} p_{1}$ is order $m-1$, noting $W \tilde{\rho}=\tilde{\alpha}_{1} \tau \tilde{\rho}$ similarly to (3.6), and $W \tau=\alpha_{\partial, 1} \tau$ by the tangency of $W$ to $\tau=0$,

$$
\begin{aligned}
\frac{1}{4} \mathrm{H}_{p}\left(a^{2}\right)= & -\left(-\hat{\rho}_{+} / 2+c_{-}^{2} \phi_{-}^{2}+\nu_{-} \phi_{-} \hat{p}_{0}-\tilde{\rho} \phi_{+}\left(W \phi_{+}\right)-\tilde{\rho} \mathrm{M} \alpha_{\partial, 1} \tau\right. \\
& \left.+\tilde{\rho} \phi_{-}\left(W \phi_{-}\right)\right) \tilde{\rho}^{-2 s}\left(\chi_{0} \chi_{0}^{\prime}\right)\left(\rho_{+}-\phi_{-}^{2}+\kappa\right) \chi\left(\rho_{+}\right)^{2} \psi\left(\tilde{\rho}^{m} p\right)^{2} \\
& +\frac{1}{4}(-2 s+m-1) \tilde{\rho}^{-2 s}\left(\tilde{\alpha}+\tilde{\rho} \tilde{\alpha}_{1}\right) \tau \chi_{0}\left(\rho_{+}-\phi_{-}^{2}+\kappa\right)^{2} \\
& \times \chi\left(\rho_{+}\right)^{2} \psi\left(\tilde{\rho}^{m} p\right)^{2} \\
& +\frac{1}{2} \tilde{\rho}^{-2 s}\left(\hat{\rho}_{+}+\tilde{\rho} W \rho_{+}\right)\left(\chi^{\prime} \chi\right)\left(\rho_{+}\right) \chi_{0}\left(\rho_{+}-\phi_{-}^{2}+\kappa\right)^{2} \psi\left(\tilde{\rho}^{m} p\right)^{2} \\
& +\frac{m}{2}\left(\tilde{\alpha}+\tilde{\rho} \tilde{\alpha}_{1}\right) \tilde{\rho}^{-2 s}\left(\tilde{\rho}^{m} p\right) \tau \chi_{0}\left(\rho_{+}-\phi_{-}^{2}+\kappa\right)^{2} \chi\left(\rho_{+}\right)^{2}\left(\psi \psi^{\prime}\right)\left(\tilde{\rho}^{m} p\right) .
\end{aligned}
$$


A key point is that the second term on the right-hand side, given by the weight $\tilde{\rho}^{-2 s+m-1}$ being differentiated, can be absorbed into the first by making $\gamma>0$ large so that $\hat{\rho}_{+} \chi_{0}^{\prime}\left(\rho_{+}-\phi_{-}^{2}+\kappa\right)$ dominates

$$
|-2 s+m-1||\tilde{\alpha}| \tau \chi_{0}\left(\rho_{+}-\phi_{-}^{2}+\kappa\right)
$$

on $\operatorname{supp} a$, which can be arranged as $|-2 s+m-1||\tilde{\alpha}| \tau$ is bounded by a sufficiently large multiple of $\hat{\rho}_{+}$there. Thus,

$$
\begin{aligned}
\frac{1}{4} \mathrm{H}_{p}\left(a^{2}\right)= & -c_{+}^{2} a_{+}^{2}-c_{-}^{2} a_{-}^{2}-a_{\partial}^{2}+2 g_{+} a_{+} \\
& +2 g_{-} a_{-}+e+\tilde{e}+2 a_{+} j_{+} p+2 a_{-} j_{-} p
\end{aligned}
$$

with

$$
\begin{aligned}
a_{ \pm}= & \tilde{\rho}^{-s} \phi_{ \pm} \sqrt{\left(\chi_{0} \chi_{0}^{\prime}\right)\left(\rho_{+}-\phi_{-}^{2}+\kappa\right)} \chi\left(\rho_{+}\right) \psi\left(\tilde{\rho}^{m} p\right), \\
a_{\partial}= & \tilde{\rho}^{-s} \tau^{1 / 2}\left(\left(\mathrm{M}\left(c_{\partial}^{2} / 2\right)-\beta_{+} \phi_{+}-\tilde{\rho} \mathrm{M} a_{\partial, 1}\right)\left(\chi_{0} \chi_{0}^{\prime}\right)\left(\rho_{+}-\phi_{-}^{2}+\kappa\right)\right. \\
& \left.-\frac{1}{4}(-2 s+m-1)\left(\tilde{\alpha}+\tilde{\rho} \tilde{\alpha}_{1}\right) \chi_{0}\left(\rho_{+}-\phi_{-}^{2}+\kappa\right)^{2}\right)^{1 / 2} \chi\left(\rho_{+}\right) \psi\left(\tilde{\rho}^{m} p\right), \\
g_{ \pm}= & \pm \frac{1}{2} \tilde{\rho}^{-s+1}\left(\left(W \phi_{ \pm}\right)-\nu_{ \pm} \tilde{\rho}^{m-1} p_{1}\right) \sqrt{\left(\chi_{0} \chi_{0}^{\prime}\right)\left(\rho_{+}-\phi_{-}^{2}+\kappa\right) \chi\left(\rho_{+}\right) \psi\left(\tilde{\rho}^{m} p\right),} \\
e= & \frac{1}{2} \tilde{\rho}^{-2 s}\left(\hat{\rho}_{+}+\hat{\rho} W \rho_{+}\right) \chi_{1}\left(\rho_{+}\right)^{2} \chi_{0}\left(\rho_{+}-\phi_{-}^{2}+\kappa\right)^{2} \psi\left(\tilde{\rho}^{m} p\right)^{2}, \\
\tilde{e}= & \frac{m}{2} \tilde{\rho}^{-2 s}\left(\tilde{\rho}^{m} p\right)\left(\tilde{\alpha}+\tilde{\rho} \tilde{\alpha}_{1}\right) \tau \chi_{0}\left(\rho_{+}-\phi_{-}^{2}+\kappa\right)^{2} \chi\left(\rho_{+}\right)^{2}\left(\psi \psi^{\prime}\right)\left(\tilde{\rho}^{m} p\right), \\
j_{ \pm}= & \pm \frac{1}{2} \nu_{ \pm} \tilde{\rho}^{-s+m} \sqrt{\left(\chi_{0} \chi_{0}^{\prime}\right)\left(\rho_{+}-\phi_{-}^{2}+\kappa\right) \chi}\left(\rho_{+}\right) \psi\left(\tilde{\rho}^{m} p\right) ;
\end{aligned}
$$

the square root in $a_{\partial}$ is that of a non-negative quantity and is $\mathcal{C}^{\infty}$ for $\mathrm{M}$ large (so that $\beta_{+} \phi_{+}$can be absorbed into $\mathrm{M}\left(c_{\partial}^{2} / 2\right)$ ) and $\gamma$ large (so that a small multiple of $\chi_{0}^{\prime}$ can be used to dominate $\left.\chi_{0}\right)$, as discussed earlier. Moreover,

$$
\begin{aligned}
& \operatorname{supp} e \subset \operatorname{supp} a, \operatorname{supp} e \cap \Gamma_{+}=\emptyset \\
& \operatorname{supp} \tilde{e} \subset \operatorname{supp} a, \operatorname{supp} \tilde{e} \cap \Sigma=\emptyset .
\end{aligned}
$$

This gives, with the various operators being Weyl quantizations of the corresponding lower case symbols,

$$
\begin{aligned}
\frac{i}{4}\left[\mathcal{P}, A^{*} A\right]= & -\left(C_{+} A_{+}\right)^{*}\left(C_{+} A_{+}\right)-\left(C_{-} A_{-}\right)^{*}\left(C_{-} A_{-}\right)-A_{\partial}^{*} A_{\partial} \\
& +G_{+}^{*} A_{+}+A_{+}^{*} G_{+}+G_{-}^{*} A_{-}+A_{-}^{*} G_{-} \\
& +E+\tilde{E}+A_{+}^{*} J_{+} \mathcal{P}+\mathcal{P}^{*} J_{+}^{*} A_{+}+A_{-}^{*} J_{-} \mathcal{P}+\mathcal{P}^{*} J_{-}^{*} A_{-}+F,
\end{aligned}
$$


where now $A \in \Psi_{\mathrm{b}}^{s-(m-1) / 2}(M), A_{ \pm}, A_{\partial} \in \Psi_{\mathrm{b}}^{s}(M), G_{ \pm} \in \Psi_{\mathrm{b}}^{s-1}(M), E \in \Psi_{\mathrm{b}}^{2 s}$ $(M), \tilde{E} \in \Psi_{\mathrm{b}}^{2 s}(M), J_{ \pm} \in \Psi_{\mathrm{b}}^{s-m}(M), F \in \Psi_{\mathrm{b}}^{2 s-2}(M)$ with $\mathrm{WF}_{\mathrm{b}}^{\prime}(F) \subset \operatorname{supp} a$.

After this point the calculations repeat the semiclassical argument: first using $\mathcal{P}-\mathcal{P}^{*} \in \Psi_{\mathrm{b}}^{m-2}(M)$,

$$
\begin{aligned}
\| C_{+} & A_{+} u\left\|^{2}+\right\| C_{-} A_{-} u\left\|^{2}+\right\| A_{\partial} u \|^{2} \\
\leq & |\langle E u, u\rangle|+|\langle\tilde{E} u, u\rangle|+|\langle A \mathcal{P} u, A u\rangle|+2\left\|A_{+} u\right\|\left\|G_{+} u\right\|+2\left\|A_{-} u\right\|\left\|G_{-} u\right\| \\
& +2\left|\left\langle J_{+} \mathcal{P} u, A_{+} u\right\rangle\right|+2\left|\left\langle J_{-} \mathcal{P} u, A_{-} u\right\rangle\right|+C_{1}\left\|\tilde{F}_{1} u\right\|_{H_{\mathrm{b}}^{s-1}}^{2}+C_{1}\|u\|_{H_{\mathrm{b}}^{-N}}^{2},
\end{aligned}
$$

where we took $\tilde{F}_{1} \in \Psi_{\mathrm{b}}^{0}(M)$ elliptic on $\mathrm{WF}_{\mathrm{b}}^{\prime}(F)$ and with $\mathrm{WF}_{\mathrm{b}}^{\prime}\left(\tilde{F}_{1}\right)$ near $\Gamma$. Noting that $\mathrm{WF}_{\mathrm{b}}^{\prime}(\tilde{E}) \cap \Sigma=\emptyset$, the elliptic estimates give

$$
|\langle\tilde{E} u, u\rangle| \leq C\left\|B_{1} \mathcal{P} u\right\|_{H_{\mathrm{b}}^{s-m}}^{2}+C\|u\|_{H_{\mathrm{b}}^{-N}}^{2}
$$

if $B_{1} \in \Psi_{\mathrm{b}}^{0}(M)$ is elliptic on supp $\tilde{e}$. Let $\Lambda \in \Psi_{\mathrm{b}}^{(m-1) / 2}(M)$ be elliptic with real principal symbol $\lambda$, and let $\Lambda^{-} \in \Psi_{\mathrm{b}}^{-(m-1) / 2}(M)$ be a parametrix for it so that $\Lambda \Lambda^{-}-\mathrm{Id}=R_{0} \in \Psi_{\mathrm{b}}^{-\infty}(M)$. Then

$$
\begin{aligned}
|\langle A \mathcal{P} u, A u\rangle| & \leq\left|\left\langle\Lambda^{-} A \mathcal{P} u, \Lambda^{*} A u\right\rangle \|+\right|\left\langle R_{0} A \mathcal{P} u, A u\right\rangle \mid \\
& \leq \frac{1}{2 \epsilon}\left\|\Lambda^{-} A \mathcal{P} u\right\|_{\mathcal{H}_{\mathrm{b}, \Gamma}^{*, 0}}^{2}+\frac{\epsilon}{2}\left\|\Lambda^{*} A u\right\|_{\mathcal{H}_{\mathrm{b}, \Gamma}^{\circ}}^{2}+C^{\prime}\|u\|_{H_{\mathrm{b}}^{-N}}^{2} .
\end{aligned}
$$

As $\Lambda^{*} A \in \Psi_{\mathrm{b}}^{s}(M)$, for sufficiently small $\epsilon>0, \frac{\epsilon}{2}\left\|\Lambda^{*} A u\right\|_{\mathcal{H}_{\mathrm{b}, \Gamma}^{0}}^{2}$ can be absorbed into $^{13}\left\|C_{+} A_{+} u\right\|^{2}+\left\|C_{-} A_{-} u\right\|^{2}+\left\|A_{\partial} u\right\|^{2}$ plus $\left\|\tilde{B}_{0} \hat{P}_{0} u\right\|_{H_{\mathrm{b}}^{s}}^{\text {s }}$, and as discussed above, the latter already has the control required for (3.11). On the other hand, taking $B_{1} \in \Psi_{\mathrm{b}}^{0}(M)$ elliptic on $\mathrm{WF}_{\mathrm{b}}^{\prime}(A)$, as $\Lambda^{-} A \in \Psi_{\mathrm{b}}^{s-m+1}(M)$,

$$
\left\|\Lambda^{-} A \mathcal{P} u\right\|_{\mathcal{H}_{\mathrm{b}, \Gamma}^{*, 0}}^{2} \leq C^{\prime \prime}\left\|B_{1} \mathcal{P} u\right\|_{\mathcal{H}_{\mathrm{b}, \Gamma}^{*, s-m+1}}^{2}+C^{\prime \prime}\|u\|_{H_{\mathrm{b}}^{-N}}^{2} .
$$

${ }^{13}$ The point being that $A_{+}^{*} C_{+}^{*} C_{+} A_{+}-\epsilon A^{*} \Lambda Q_{+}^{*} Q_{+} \Lambda^{*} A$ has principal symbol $c_{+}^{2} a_{+}^{2}-\epsilon a^{2} \phi_{+}^{2} \lambda^{2}$ which can be written as the square of a real symbol for $\epsilon>0$ small in view of the main difference in vanishing factors in the two terms being that $\chi_{0}^{\prime}$ in $a_{+}^{2}$ is replaced by $\chi_{0}$ in $a$, and thus the corresponding operator can be expressed as $\tilde{C}^{*} \tilde{C}$ for suitable $\tilde{C}$, modulo an element of $\Psi_{\mathrm{b}}^{2 s-2}(M)$, with the latter contributing to the $H_{\mathrm{b}}^{s-1}$ error term on the right-hand side of (3.11). 
Similarly, to deal with the $J_{ \pm}$terms on the right-hand side of (3.18), one writes

$$
\begin{aligned}
\left|\left\langle J_{ \pm} \mathcal{P} u, A_{ \pm} u\right\rangle\right| & \leq \frac{1}{2 \epsilon}\left(\left\|B_{1} \mathcal{P} u\right\|_{H_{\mathrm{b}}^{s-m}}^{2}+C^{\prime \prime}\|u\|_{H_{\mathrm{b}}^{-N}}^{2}\right)+\frac{\epsilon}{2}\left\|A_{ \pm} u\right\|_{L^{2}}^{2} \\
& \leq \frac{1}{2 \epsilon}\left(\left\|B_{1} \mathcal{P} u\right\|_{\mathcal{H}_{\mathrm{b}, \Gamma}^{*, s-m}}^{2}+C^{\prime \prime}\|u\|_{H_{\mathrm{b}}^{-N}}^{2}\right)+\frac{\epsilon}{2}\left\|A_{ \pm} u\right\|_{L^{2}}^{2}
\end{aligned}
$$

while the $G_{ \pm}$terms can be estimated by

$$
\epsilon\left\|A_{+} u\right\|^{2}+\epsilon^{-1}\left\|G_{+} u\right\|^{2}+\epsilon\left\|A_{-} u\right\|^{2}+\epsilon^{-1}\left\|G_{-} u\right\|^{2},
$$

and for $\epsilon>0$ sufficiently small, the $\left\|A_{ \pm} u\right\|^{2}$ terms in both cases can be absorbed into the left-hand side of (3.18) while the $G_{ \pm}$into the error term. This gives, with $\tilde{F}_{2}$ having properties as $\tilde{F}_{1}$,

$$
\begin{aligned}
& \left\|C_{+} A_{+} u\right\|^{2}+\left\|C_{-} A_{-} u\right\|^{2}+\left\|A_{\partial} u\right\|^{2} \\
& \quad \leq|\langle E u, u\rangle|+C\left\|B_{1} \mathcal{P} u\right\|_{\mathcal{H}_{\mathrm{b}, \Gamma}^{*, s-m+1}}^{2}+C_{2}\left\|\tilde{F}_{2} u\right\|_{H_{\mathrm{b}}^{s-1}}^{2}+C_{2}\|u\|_{H_{\mathrm{b}}^{-N}}^{2} .
\end{aligned}
$$

By the remark before the statement of the theorem, if $B_{0} \in \Psi_{\mathrm{b}}^{0}(M)$ is such that $\chi_{0}\left(\rho_{+}-\phi_{-}^{2}+\kappa\right) \chi\left(\rho_{+}\right) \psi(p)>0$ on $\mathrm{WF}_{\mathrm{b}}^{\prime}\left(B_{0}\right),\left\|B_{0} u\right\|_{H_{\mathrm{b}}^{s-1 / 2}}^{2}$ can be added to the left-hand side at the cost of changing the constant in front of $\left\|\tilde{F}_{2} u\right\|_{H_{\mathrm{b}}^{s-1}}^{2}+\|u\|_{H_{\mathrm{b}}^{-N}}^{2}$ on the right-hand side. Taking such $B_{0} \in \Psi_{\mathrm{b}}^{0}(M)$, and $B_{1}$ elliptic on $\mathrm{WF}_{\mathrm{b}}^{\prime}(A)$ as before, $B_{2} \in \Psi_{\mathrm{b}}^{0}(M)$ elliptic on $\mathrm{WF}_{\mathrm{b}}^{\prime}(E)$ but with $\mathrm{WF}_{\mathrm{b}}^{\prime}\left(B_{2}\right)$ disjoint from $\Gamma_{+}$, we conclude that

$$
\left\|B_{0} u\right\|_{\mathcal{H}_{\mathrm{b}, \Gamma}^{s}}^{2} \leq C\left\|B_{1} \mathcal{P} u\right\|_{\mathcal{H}_{\mathrm{b}, \Gamma}^{*, s-m+1}}^{2}+C\left\|B_{2} u\right\|_{H_{\mathrm{b}}^{s}}^{2}+C\left\|\tilde{F}_{2} u\right\|_{H_{\mathrm{b}}^{s-1}}^{2}+C\|u\|_{H_{\mathrm{b}}^{-N}}^{2},
$$

proving (3.11), up to redefining $B_{j}$ by multiplication by a positive constant. Recall that unless one makes sufficient a priori assumptions on the regularity of $u$, one actually needs to regularize, but as mentioned after (3.14), the regularizer is handled in exactly the same manner as the weight.

Now in general, with $\chi$ as before, but supported in $[0,1]$ instead of $[0, R]$, let $\chi_{R}=\chi(\cdot / R)$ and write $a=a_{R, \kappa}$ to emphasize its dependence on these quantities. When $R$ and $\kappa$ are decreased, supp $a_{R, \kappa}$ also decreases in $\Sigma$ in the strong sense that $0<R<R^{\prime}$ and $0<\kappa<\kappa^{\prime}$ imply that $a_{R^{\prime}, \kappa^{\prime}}$ is elliptic on $\operatorname{supp} a_{R, \kappa}$ within $\Sigma$, and indeed globally if the cutoff $\psi$ is suitably adjusted as well. Thus, if $u \in H_{\mathrm{b}}^{-N}$, say, one uses first (3.11) with $s=-N+1$, and with $B_{j}$ given by the proof above, so the $B_{3} u$ term is a priori bounded, to conclude that $B_{0} u \in \mathcal{H}_{\mathrm{b}, \Gamma}^{s}$ and the estimate holds, so in particular, $u$ is in $H_{\mathrm{b}}^{-N+1 / 2}$ microlocally near $\Gamma$ (concretely, on the elliptic set of $B_{0}$ ). Now one 
decreases $\kappa$ and $R$ by an arbitrarily small amount and applies (3.11) with $s=-N+3 / 2$; the $B_{3} u$ term is now a priori bounded by the microlocal membership of $u$ in $H_{\mathrm{b}}^{-N+1 / 2}$, and one concludes that $B_{0} u \in \mathcal{H}_{\mathrm{b}, \Gamma}^{-N+3 / 2}$, so in particular $u$ is microlocally in $H_{\mathrm{b}}^{-N+1}$. Proceeding inductively, one deduces the first statement of the theorem, (3.8).

If one reverses the role of $\Gamma_{+}$and $\Gamma_{-}$in the statement of the theorem, one simply reverses the roles of $\rho_{+}=\phi_{+}^{2}+\mathrm{M} \tau$ and $\phi_{-}^{2}$ in the definition of $a$ in (3.13). This reverses the signs of all terms on the right-hand side of (3.15) whose sign mattered below, and thus the signs of the first three terms on the right-hand side of (3.17), which then does not affect the rest of the argument.

In order to prove (3.9), one simply adds a factor $\tau^{-2 r}$ to the definition of $a$ in (3.13). This adds a factor $\tau^{-2 r}$ to every term on the right-hand side of (3.17), as well as an additional term

$$
\frac{r}{2} \tau^{-2 r} \tilde{\rho}^{-2 s} c_{\partial}^{2} \chi_{0}\left(\rho_{+}-\phi_{-}^{2}+\kappa\right)^{2} \chi\left(\rho_{+}\right)^{2} \psi(p)^{2},
$$

which for $r<0$ has the same sign as the terms whose sign was used above, and indeed can be written as the negative of a square. Thus (3.16) becomes

$$
\begin{aligned}
\frac{1}{4} \mathrm{H}_{p}\left(a^{2}\right)= & -c_{+}^{2} a_{+}^{2}-c_{-}^{2} a_{-}^{2}-a_{\partial}^{2}-a_{r}^{2} \\
& +2 g_{+} a_{+}+2 g_{-} a_{-}+e+\tilde{e}+2 j_{+} a_{+} p+2 j_{-} a_{-} p
\end{aligned}
$$

with

$$
a_{r}=\sqrt{\frac{-r}{2}} \tau^{-r} \tilde{\rho}^{-s} c_{\partial} \chi_{0}\left(\rho_{+}-\phi_{-}^{2}+\kappa\right) \chi\left(\rho_{+}\right) \psi(p),
$$

and all other terms as above apart from the additional factor of $\tau^{-r}$ in the definition of $a_{ \pm}$, etc. Since $a_{r}$ is actually elliptic at $\Gamma$ when $r \neq 0$, this proves the desired estimate (and one does not need to use the improved properties given by the Weyl calculus!).

When the role of $\Gamma_{+}$and $\Gamma_{-}$is reversed, there is an overall sign change, and thus $r>0$ gives the advantageous sign; the rest of the argument is unchanged.

Remark 3.4. As in the semiclassical setting, see Remark 2.1, the estimate (3.8) can be strengthened by adding the term $\left\|B_{0} \hat{P}_{0} u\right\|_{H_{\mathrm{b}}^{s+1}}$ to the left-hand side, which is controlled by elliptic regularity, likewise for (3.9) and (3.10). A more natural way of phrasing such an improvement is to use "coisotropic, normally isotropic" spaces $\tilde{\mathcal{H}}_{\mathrm{b}, \Gamma}^{s}$ and $\tilde{\mathcal{H}}_{\mathrm{b}, \Gamma}^{*, s}$ in the estimate $(3.8)$, where the 
squared norm on $\tilde{\mathcal{H}}_{\mathrm{b}, \Gamma}^{s}$ is defined by

$$
\begin{aligned}
\|u\|_{\tilde{\mathcal{H}}_{\mathrm{b}, \Gamma}^{s}}^{2}= & \left\|Q_{0} u\right\|_{H_{\mathrm{b}}^{s}}^{2}+\left\|Q_{+} u\right\|_{H_{\mathrm{b}}^{s}}^{2}+\left\|Q_{-} u\right\|_{H_{\mathrm{b}}^{s}}^{2}+\left\|\tau^{1 / 2} u\right\|_{H_{\mathrm{b}}^{s}}^{2} \\
& +\left\|\hat{P}_{0} u\right\|_{H_{\mathrm{b}}^{s+1 / 2}}^{2}+\|u\|_{H_{\mathrm{b}}^{s-1 / 2}}^{2}
\end{aligned}
$$

i.e., strengthening the norm of $\hat{P}_{0} u$ by a half, which strengthens the space and weakens its dual. To obtain the necessary elliptic estimate (3.12) with the strengthened norms on the terms involving $\tilde{B}_{0}$, but keeping the norm on $\tilde{B}_{3} u$ (which is required for the iterative argument at the end of the proof), one can choose $\tilde{B}_{0}$ with $\mathrm{WF}_{\mathrm{b}}^{\prime}\left(I-\tilde{B}_{0}\right) \cap \Gamma=\emptyset$ so that $\tilde{B}_{3}$ can be chosen to be microsupported away from $\Gamma$, and thus $\left\|\tilde{B}_{3} u\right\|_{H_{\mathrm{b}}^{s-1 / 2}} \leq C\left\|\tilde{B}_{3} u\right\|_{\mathcal{H}_{\mathrm{b}, \Gamma}^{s-1 / 2}}$ is controlled using (3.8), with the norm on $B_{1} \mathcal{P} u$ being $\left\|B_{1} \mathcal{P} u\right\|_{\mathcal{H}_{\mathrm{b} \Gamma}^{*, s-m+1 / 2}} \leq$ $C\left\|B_{1} \mathcal{P} u\right\|_{\tilde{\mathcal{H}}_{\mathrm{b}, \Gamma}^{*, s-m+1}}$, and the error term being measured in $H_{\mathrm{b}}^{s-3 / 2} \supset H_{\mathrm{b}}^{s-1}$, as required.

\section{Acknowledgement}

The authors were supported in part by A.V.'s National Science Foundation grants DMS-0801226 and DMS-1068742 and P.H. was supported in part by a Gerhard Casper Stanford Graduate Fellowship and the German National Academic Foundation. They are grateful to the referee for comments that significantly improved the exposition in the manuscript.

\section{References}

[1] J.-F. Bony, N. Burq and T. Ramond, Minoration de la résolvante dans le cas captif, C. R. Math., 348(23-24) (2010), 1279-1282.

[2] K. Datchev and A. Vasy, Propagation through trapped sets and semiclassical resolvent estimates, Ann. Inst. Fourier, 62(6) (2012), 2347-2377.

[3] K. Datchev and A. Vasy, Semiclassical resolvent estimates at trapped sets, Ann. Inst. Fourier, 62(6) (2012), 2379-2384.

[4] S. Dyatlov, Asymptotics of linear waves and resonances with applications to black holes, Preprint, 2013, arXiv:1305.1723.

[5] S. Dyatlov, Spectral gaps for normally hyperbolic trapping, Preprint, 2014, arXiv: 1403.6401. 
[6] P. Hintz and A. Vasy, Global analysis of nonlinear wave equations on asymptotically Kerr-de Sitter spaces, Preprint, 2014, arXiv: 1404.1348.

[7] R.B. Melrose, The Atiyah-Patodi-Singer index theorem, 4 Research Notes in Mathematics, A K Peters Ltd., Wellesley, MA, 1993.

[8] R.B. Melrose, A. Vasy and J. Wunsch, Diffraction of singularities for the wave equation on manifolds with corners, Astérisque, 351 (2013), $\mathrm{vi}+136 \mathrm{pp}$.

[9] S. Nonnenmacher and M. Zworski, Decay of correlations for normally hyperbolic trapping, Preprint, 2013, arXiv:1302.4483.

[10] A. Vasy, Microlocal analysis of asymptotically hyperbolic and Kerr-de Sitter spaces, With an appendix by S. Dyatlov. Invent. Math., 194 (2013), 381-513.

[11] J. Wunsch and M. Zworski, Erratum to "Resolvent estimates for normally hyperbolic trapped sets", Posted on http://www.math.northwestern.edu/ jwunsch/erratum_wz.pdf

[12] J. Wunsch and M. Zworski, Resolvent estimates for normally hyperbolic trapped sets, Ann. Henri Poincaré, 12(7) (2011), 1349-1385.

[13] M. Zworski, Semiclassical analysis, Graduate studies in Mathematics, American Mathematical Society, 2012.

Department OF MAThematics

STANFORD UNIVERSITY

STANFORD, CA 94305-2125

USA

E-mail address: phintz@math.stanford.edu

E-mail address: andras@math.stanford.edu

Received November 27, 2013 